\title{
COMBINATORIAL HOPF ALGEBRAS FROM PROS
}

\author{
JEAN-PAUL BULTEL AND SAMUELE GIRAUDO
}

\begin{abstract}
We introduce a general construction that takes as input a so-called stiff PRO and that outputs a Hopf algebra. Stiff PROs are particular PROs that can be described by generators and relations with precise conditions. Our construction generalizes the classical construction from operads to Hopf algebras of van der Laan. We study some of its properties and review some examples of application. We get in particular Hopf algebras on heaps of pieces and retrieve some deformed versions of the noncommutative Faà di Bruno algebra introduced by Foissy.
\end{abstract}

\section{Contents}

Introduction

1. Algebraic structures and background

1.1. Combinatorial Hopf algebras

1.2. The natural Hopf algebra of an operad

1.3. PROs and free PROs

2. From PROs to combinatorial Hopf algebras 9

2.1. The Hopf algebra of a free PRO 9

2.2. Properties of the construction 12

2.3. The Hopf algebra of a stiff PRO 15

2.4. Related constructions 20

3. Examples of application of the construction 22

3.1. Hopf algebras of forests 22

3.2. The Faà di Bruno algebra and its deformations 25

3.3. Hopf algebra of forests of bitrees 27

3.4. Hopf algebra of heaps of pieces 29

3.5. Hopf algebra of heaps of friable pieces 31

Concluding remarks and perspectives 33

References

Date: August 21, 2018.

2010 Mathematics Subject Classification. 05E99, 05E05, 05C05, 57T05, $18 \mathrm{D} 50$.

Key words and phrases. Operad; PRO; Hopf algebra; Noncommutative symmetric function; Faà di Bruno algebra.

Phone number and email address of the corresponding author: +33160957558, samuele.giraudo@u-pem.fr. 


\section{INTRODUCTION}

Operads are algebraic structures introduced in the 70s by Boardman and Vogt [BV73] and by May [May72] in the context of algebraic topology to offer a formalization of the notion of operators and their composition (see [Mar08] and [LV12] for a very complete presentation of the theory of operads). Operads provide therefore a unified framework to study some sorts of a priori very different algebras, such as associative algebras, Lie algebras, and commutative algebras. Besides, the theory of operads is also beneficial in combinatorics [Cha08] since it provides some ways to decompose combinatorial objects into elementary pieces. On the other hand, the theory of Hopf algebras holds a special place in algebraic combinatorics [JR79]. In recent years, many Hopf algebras were defined and studied, and most of these involve very famous combinatorial objects such as permutations [MR95,DHT02], standard Young tableaux [PR95,DHT02], or binary trees [LR98, HNT05].

These two theories - operads and Hopf algebras - have several interactions. One of these is a construction [vdL04] taking an operad $\mathcal{O}$ as input and producing a bialgebra $H(\mathcal{O})$ as output, which is called the natural bialgebra of $\mathcal{O}$. This construction has been studied in some recent works: in [CL07], it is shown that $H$ can be rephrased in terms of an incidence Hopf algebra of a certain family of posets, and in [ML14], a general formula for its antipode is established. Let us also cite [Fra08] in which this construction is considered to study series of trees.

The initial motivation of our work was to generalize this $H$ construction with the aim of constructing some new and interesting Hopf algebras. The direction we have chosen is to start with PROs, algebraic structures which generalize operads in the sense that PROs deal with operators with possibly several outputs. Surprisingly, these structures appeared earlier than operads in the work of Mac Lane [ML65]. Intuitively, a PRO $\mathcal{P}$ is a set of operators together with two operations: an horizontal composition and a vertical composition. The first operation takes two operators $x$ and $y$ of $\mathcal{P}$ and builds a new one whose inputs (resp. outputs) are, from left to right, those of $x$ and then those of $y$. The second operation takes two operators $x$ and $y$ of $\mathcal{P}$ and produces a new one obtained by plugging the outputs of $y$ onto the inputs of $x$. Basic and modern references about PROs are [Lei04] and [Mar08].

Our main contribution consists in the definition of a new construction $\mathrm{H}$ from PROs to bialgebras. Roughly speaking, the construction $\mathrm{H}$ can be described as follows. Given a PRO $\mathcal{P}$ satisfying some mild properties, the bialgebra $\mathrm{H} \mathcal{P}$ has bases indexed by a particular subset of elements of $\mathcal{P}$. The product of $\mathrm{H} \mathcal{P}$ is the horizontal composition of $\mathcal{P}$ and the coproduct of $\mathrm{H} \mathcal{P}$ is defined from the vertical composition of $\mathcal{P}$, enabling to separate a basis element into two smaller parts. The properties satisfied by $\mathcal{P}$ imply, in a nontrivial way, that the product and the coproduct of $\mathrm{HP}$ satisfy the required axioms to be a bialgebra. This construction generalizes $H$ and establishes a new connection between the theory of PROs and the theory of Hopf algebras.

Our results are organized as follows. In Section 1, we recall some general background about Hopf algebras, operads, and PROs. In particular, we give a description of free PROs in terms of prographs, similar to the one of Lafont [Laf11]. We also recall the natural bialgebra construction of an operad. We provide in Section 2 the description of our new construction $\mathrm{H}$. A first version of this construction is presented, associating a bialgebra $\mathrm{H}(\mathcal{P})$ with a free $\mathrm{PRO} \mathcal{P}$. We then present an extended version of the construction, taking as input non-necessarily free PROs 
satisfying some properties, called stiff PROs. Next, we consider two well-known constructions of PROs [Mar08], one, R, taking as input operads and the other, B, taking as input monoids. We prove that under some mild conditions, these constructions produce stiff PROs. We establish that the natural bialgebra of an operad can be reformulated as a particular case of our construction $\mathrm{H}$. We conclude by giving some examples of application of $\mathrm{H}$ in Section 3. The Hopf algebras that we obtain are very similar to the Connes-Kreimer Hopf algebra [CK98] in the sense that their coproduct can be computed by means of admissible cuts in various combinatorial objects. From very simple stiff PROs, we reconstruct the Hopf algebra of noncommutative symmetric functions Sym [GKL ${ }^{+}$95,KLT97] and the noncommutative Fàa di Bruno algebra FdB $_{1}$ [BFK06]. Besides, we present a way of using $\mathrm{H}$ to reconstruct some of the Hopf algebras $\mathbf{F d B}_{\gamma}$, a $\gamma-$ deformation of $\mathbf{F d B}_{1}$ introduced by Foissy [Foi08]. We also obtain several other Hopf algebras, which, respectively, involve forests of planar rooted trees, some kind of graphs consisting of nodes with one parent and several children or several parents and one child that we call forests of bitrees, heaps of pieces (see [Vie86] for a general presentation of these combinatorial objects), and a particular class of heaps of pieces that we call heaps of friable pieces. All these Hopf algebras depend on a nonnegative integer as parameter.

Acknowledgments. The authors would like to thank Jean-Christophe Novelli for his suggestions during the preparation of this paper. Moreover, the authors would like to thank the anonymous referee for its useful suggestions, improving the paper. The computations of this work have been done with the open-source mathematical software Sage $\left[\mathrm{S}^{+} 14\right]$ and one of its extensions, Sage-Combinat [SCc08].

Notations. For any integer $n \geqslant 0,[n]$ denotes the set $\{1, \ldots, n\}$. If $u$ is a word and $i$ is a positive integer no greater than the length of $u, u_{i}$ denotes the $i$-th letter of $u$. The empty word is denoted by $\epsilon$.

\section{Algebraic structures And BaCkground}

We recall in this preliminary section some basics about the algebraic structures in play in all this work, i.e., Hopf algebras, operads, and PROs. We also present some well-known Hopf algebras and recall the construction associating a combinatorial Hopf algebra with an operad.

1.1. Combinatorial Hopf algebras. In the sequel, all vector spaces have $\mathbb{C}$ as ground field. By algebra we mean a unitary associative algebra and by coalgebra a counitary coassociative coalgebra. We call combinatorial Hopf algebra any graded bialgebra $\mathcal{H}=\bigoplus_{n \geqslant 0} \mathcal{H}_{n}$ such that for any $n \geqslant 1$, the $n$-th homogeneous component $\mathcal{H}_{n}$ of $\mathcal{H}$ has finite dimension and the dimension of $\mathcal{H}_{0}$ is 1 . The degree of any element $x \in \mathcal{H}_{n}$ is $n$ and is denoted by $\operatorname{deg}(x)$. Combinatorial Hopf algebras are Hopf algebras because the antipode can be defined recursively degree by degree. Let us now review some classical combinatorial Hopf algebras which play an important role in this work. 
1.1.1. Fà̀ di Bruno algebra and its deformations. Let $F d B$ be the free commutative algebra generated by elements $h_{n}, n \geqslant 1$ with $\operatorname{deg}\left(h_{n}\right)=n$. The bases of $F d B$ are thus indexed by integer partitions, and the unit is denoted by $h_{0}$. This is the algebra of symmetric functions [Mac95]. There are several ways to endow $F d B$ with a coproduct to turn it into a Hopf algebra. In [Foi08], Foissy obtains, as a byproduct of his investigation of combinatorial Dyson-Schwinger equations in the Connes-Kreimer algebra, a one-parameter family $\Delta_{\gamma}, \gamma \in \mathbb{R}$, of coproducts on $F d B$, defined by using alphabet transformations (see [Mac95]), by

$$
\Delta_{\gamma}\left(h_{n}\right):=\sum_{k=0}^{n} h_{k} \otimes h_{n-k}((k \gamma+1) X),
$$

where, for any $\alpha \in \mathbb{R}$ and $n \in \mathbb{N}, h_{n}(\alpha X)$ is the coefficient of $t^{n}$ in $\left(\sum_{k \geqslant 0} h_{k} t^{k}\right)^{\alpha}$. In particular,

$$
\Delta_{0}\left(h_{n}\right)=\sum_{k=0}^{n} h_{k} \otimes h_{n-k}
$$

The algebra $F d B$ with the coproduct $\Delta_{0}$ is the classical Hopf algebra of symmetric functions Sym [Mac95]. Moreover, for all $\gamma \neq 0$, all $F d B_{\gamma}$ are isomorphic to $F d B_{1}$, which is known as the Faà di Bruno algebra [JR79]. The coproduct $\Delta_{0}$ comes from the interpretation of $F d B$ as the algebra of polynomial functions on the multiplicative group $\left(G(t):=\left\{1+\sum_{k \geqslant 1} a_{k} t^{k}\right\}, \cdot\right)$ of formal power series of constant term 1 , and $\Delta_{1}$ comes from its interpretation as the algebra of polynomial functions on the group $(t G(t), \circ)$ of formal diffeomorphisms of the real line.

1.1.2. Noncommutative analogs. Formal power series in one variable with coefficients in a noncommutative algebra can be composed (by substitution of the variable). This operation is not associative, so that they do not form a group. For example, when $a$ and $b$ belong to a noncommutative algebra, one has

$$
\left(t^{2} \circ a t\right) \circ b t=a^{2} t^{2} \circ b t=a^{2} b^{2} t^{2}
$$

but

$$
t^{2} \circ(a t \circ b t)=t^{2} \circ a b t=a b a b t^{2} .
$$

However, the analogue of the Faà di Bruno algebra still exists in this context and is known as the noncommutative Faà di Bruno algebra. It is investigated in [BFK06] in view of applications in quantum field theory. In [Foi08], Foissy also obtains an analogue of the family $F d B_{\gamma}$ in this context. Indeed, considering noncommutative generators $\mathbf{S}_{n}$ (with $\operatorname{deg}\left(\mathbf{S}_{n}\right)=n$ ) instead of the $h_{n}$, for all $n \geqslant 1$, leads to a free noncommutative algebra FdB whose bases are indexed by integer compositions. This is the algebra of noncommutative symmetric functions [GKL $\left.{ }^{+} 95\right]$. The addition of the coproduct $\Delta_{\gamma}$ defined by

$$
\Delta_{\gamma}\left(\mathbf{S}_{n}\right):=\sum_{k=0}^{n} \mathbf{S}_{k} \otimes \mathbf{S}_{n-k}((k \gamma+1) A),
$$

where, for any $\alpha \in \mathbb{R}$ and $n \in \mathbb{N}, \mathbf{S}_{n}(\alpha A)$ is the coefficient of $t^{n}$ in $\left(\sum_{k \geqslant 0} \mathbf{S}_{k} t^{k}\right)^{\alpha}$, forms a noncommutative Hopf algebra $\mathbf{F d B}_{\gamma}$. In particular,

$$
\Delta_{0}\left(\mathbf{S}_{n}\right)=\sum_{k=0}^{n} \mathbf{S}_{k} \otimes \mathbf{S}_{n-k},
$$


where $\mathbf{S}_{0}$ is the unit. In this way, $\mathbf{F d B}$ with the coproduct $\Delta_{0}$ is the Hopf algebra of noncommutative symmetric functions $\mathbf{S y m}$ [GKL ${ }^{+} 95, \mathrm{KLT} 97$ ], and for all $\gamma \neq 0$, all the $\mathbf{F d B}_{\gamma}$ are isomorphic to $\mathbf{F d B}_{1}$, which is the noncommutative Faà di Bruno algebra.

1.2. The natural Hopf algebra of an operad. We shall consider in this work only nonsymmetric operads in the category of sets. For this reason, we shall call these simply operads.

1.2.1. Operads. In our context, an operad is a triple $\left(\mathcal{O}, \circ_{i}, \mathbb{1}\right)$ where

$$
\mathcal{O}:=\bigsqcup_{n \geqslant 1} \mathcal{O}(n)
$$

is a graded set,

$$
\circ_{i}: \mathcal{O}(n) \times \mathcal{O}(m) \rightarrow \mathcal{O}(n+m-1), \quad n, m \geqslant 1, i \in[n],
$$

is a composition map, called partial composition, and $\mathbb{1} \in \mathcal{O}(1)$ is a unit. These data have to satisfy the relations

$$
\begin{gathered}
\left(x \circ_{i} y\right) \circ_{i+j-1} z=x \circ_{i}\left(y \circ_{j} z\right), \quad x \in \mathcal{O}(n), y \in \mathcal{O}(m), z \in \mathcal{O}(k), i \in[n], j \in[m], \\
\left(x \circ_{i} y\right) \circ_{j+m-1} z=\left(x \circ_{j} z\right) \circ_{i} y, \quad x \in \mathcal{O}(n), y \in \mathcal{O}(m), z \in \mathcal{O}(k), 1 \leqslant i<j \leqslant n, \\
\mathbb{1} \circ_{1} x=x=x \circ_{i} \mathbb{1}, \quad x \in \mathcal{O}(n), i \in[n] .
\end{gathered}
$$

Besides, we shall denote by

$$
\circ: \mathcal{O}(n) \times \mathcal{O}\left(m_{1}\right) \times \cdots \times \mathcal{O}\left(m_{n}\right) \rightarrow \mathcal{O}\left(m_{1}+\cdots+m_{n}\right), \quad n, m_{1}, \ldots, m_{n} \geqslant 1,
$$

the total composition map of $\mathcal{O}$. It is defined for any $x \in \mathcal{O}(n)$ and $y_{1}, \ldots, y_{n} \in \mathcal{O}$ by

$$
x \circ\left[y_{1}, \ldots, y_{n}\right]:=\left(\ldots\left(\left(x \circ_{n} y_{n}\right) \circ_{n-1} y_{n-1}\right) \ldots\right) \circ_{1} y_{1} .
$$

If $x$ is an element of $\mathcal{O}(n)$, we say that the arity $|x|$ of $x$ is $n$. An operad morphism is a map $\phi: \mathcal{O}_{1} \rightarrow \mathcal{O}_{2}$ between two operads $\mathcal{O}_{1}$ and $\mathcal{O}_{2}$ such that $\phi$ commutes with the partial composition maps and preserves the arities. A subset $S$ of an operad $\mathcal{O}$ is a suboperad of $\mathcal{O}$ if $\mathbb{1} \in S$ and the composition of $\mathcal{O}$ is stable in $S$. The suboperad of $\mathcal{O}$ generated by a subset $G$ of $\mathcal{O}$ is the smallest suboperad of $\mathcal{O}$ containing $G$.

1.2.2. The natural bialgebra of an operad. Let us recall a very simple construction associating a Hopf algebra with an operad. A slightly different version of this construction is considered in [vdL04, CL07, ML14]. Let $\mathcal{O}$ be an operad and denote by $\mathcal{O}^{+}$the set $\mathcal{O} \backslash\{\mathbb{1}\}$. The natural bialgebra of $\mathcal{O}$ is the free commutative algebra $H(\mathcal{O})$ spanned by the $T_{x}$, where the $x$ are elements of $\mathcal{O}^{+}$. The bases of $H(\mathcal{O})$ are thus indexed by finite multisets of elements of $\mathcal{O}^{+}$. The unit of $H(\mathcal{O})$ is denoted by $T_{\mathbb{1}}$ and the coproduct of $H(\mathcal{O})$ is the unique algebra morphism satisfying, for any element $x$ of $\mathcal{O}^{+}$,

$$
\Delta\left(T_{x}\right)=\sum_{\substack{y, z_{1}, \ldots, z_{\ell} \in \mathcal{O} \\ y \circ\left[z_{1}, \ldots, z_{\ell}\right]=x}} T_{y} \otimes T_{z_{1}} \ldots T_{z_{\ell}} .
$$


The bialgebra $H(\mathcal{O})$ can be graded by $\operatorname{deg}\left(T_{x}\right):=|x|-1$. Note that with this grading, when $\mathcal{O}(1)=\{\mathbb{1}\}$ and the $\mathcal{O}(n)$ are finite for all $n \geqslant 1, H(\mathcal{O})$ becomes a combinatorial Hopf algebra.

1.3. PROs and free PROs. We recall here the definitions of PROs and free PROs in terms of prographs and introduce the notions of reduced and indecomposable elements, which will be used in the following sections.

1.3.1. PROs. A $P R O$ is a quadruple $\left(\mathcal{P}, *, \circ, \mathbb{1}_{p}\right)$ where $\mathcal{P}$ is a bigraded set of the form

$$
\mathcal{P}:=\bigsqcup_{p \geqslant 0} \bigsqcup_{q \geqslant 0} \mathcal{P}(p, q)
$$

such that for any $p, q \geqslant 0, \mathcal{P}(p, q)$ contains elements $x$ with $\mathrm{i}(x):=p$ as input arity and $\mathrm{o}(x):=q$ as output arity, $*$ is a map of the form

$$
\text { * }: \mathcal{P}(p, q) \times \mathcal{P}\left(p^{\prime}, q^{\prime}\right) \rightarrow \mathcal{P}\left(p+p^{\prime}, q+q^{\prime}\right), \quad p, p^{\prime}, q, q^{\prime} \geqslant 0,
$$

called horizontal composition, $\circ$ is a map of the form

$$
\circ: \mathcal{P}(q, r) \times \mathcal{P}(p, q) \rightarrow \mathcal{P}(p, r), \quad p, q, r \geqslant 0,
$$

called vertical composition, and for any $p \geqslant 0, \mathbb{1}_{p}$ is an element of $\mathcal{P}(p, p)$ called unit of arity $p$.

These data have to satisfy for all $x, y, z \in \mathcal{P}$ the six relations

$$
\begin{gathered}
(x * y) * z=x *(y * z), \quad x, y, z \in \mathcal{P}, \\
(x \circ y) \circ z=x \circ(y \circ z), \quad x, y, z \in \mathcal{P}, \mathrm{i}(x)=\mathrm{o}(y), \mathrm{i}(y)=\mathrm{o}(z), \\
(x \circ y) *\left(x^{\prime} \circ y^{\prime}\right)=\left(x * x^{\prime}\right) \circ\left(y * y^{\prime}\right), \quad x, x^{\prime}, y, y^{\prime} \in \mathcal{P}, \mathrm{i}(x)=\mathrm{o}(y), \mathrm{i}\left(x^{\prime}\right)=\mathrm{o}\left(y^{\prime}\right), \\
\mathbb{1}_{p} * \mathbb{1}_{q}=\mathbb{1}_{p+q}, \quad p, q \geqslant 0, \\
x * \mathbb{1}_{0}=x=\mathbb{1}_{0} * x, \quad x \in \mathcal{P}, \\
x \circ \mathbb{1}_{p}=x=\mathbb{1}_{q} \circ x, \quad x \in \mathcal{P}, p, q \geqslant 0, \mathrm{i}(x)=p, \mathrm{o}(x)=q .
\end{gathered}
$$

A PRO morphism is a map $\phi: \mathcal{P}_{1} \rightarrow \mathcal{P}_{2}$ between two PROs $\mathcal{P}_{1}$ and $\mathcal{P}_{2}$ such that $\phi$ commutes with the horizontal and vertical compositions and preserves the input and output arities. A subset $S$ of a PRO $\mathcal{P}$ is a sub-PRO of $\mathcal{P}$ if $\mathbb{1}_{p} \in S$ for any $p \geqslant 0$ and the horizontal and vertical compositions of $\mathcal{P}$ are stable in $S$. The sub-PRO of $\mathcal{P}$ generated by a subset $G$ of $\mathcal{P}$ is the smallest sub-PRO of $\mathcal{P}$ containing $G$. An equivalence relation $\equiv$ on $\mathcal{P}$ is a congruence of PROs if all the elements of a same $\equiv$-equivalence class have the same input arity and the same output arity, and $\equiv$ is compatible with the horizontal and the vertical composition. Any congruence $\equiv$ of $\mathcal{P}$ gives rise to a $P R O$ quotient of $\mathcal{P}$ denoted by $\mathcal{P} / \equiv$ and defined in the expected way. 
1.3.2. Free PROs. Let us now set our terminology about free PROs and its elements in terms of prographs. From now,

$$
G:=\bigsqcup_{p \geqslant 1} \bigsqcup_{q \geqslant 1} G(p, q)
$$

is a bigraded set. An elementary prograph e over $G$ is a formal operator labeled by an element a of $G(p, q)$. The input (resp. output) arity of $e$ is $p$ (resp. q). We represent $e$ as a rectangle labeled by $g$ with $p$ incoming edges (below the rectangle) and $q$ outgoing edges (above the rectangle). For instance, if $\mathrm{a} \in G(2,3)$, the elementary prograph labeled by a is depicted by

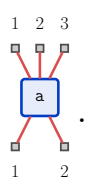

A prograph over $G$ is a formal operator defined recursively as follows. A prograph over $G$ can be either an elementary prograph over $G$, or a special element, the wire depicted by

$$
\text { ! }
$$

or a combination of two prographs over $G$ through the following two operations. The first one, denoted by $*$, consists in placing two prographs side by side. For instance, if $x$ is a prograph with $p$ inputs (resp. $q$ outputs) and $y$ is a prograph with $p^{\prime}$ inputs (resp. $q^{\prime}$ outputs),

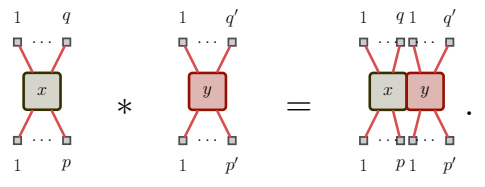

The second one, denoted by $\circ$, consists in connecting the inputs of a first prograph over the outputs of a second. For instance, if $x$ is a prograph with $p$ inputs (resp. $q$ outputs) and $y$ is a prograph with $r$ inputs (resp. $p$ outputs),

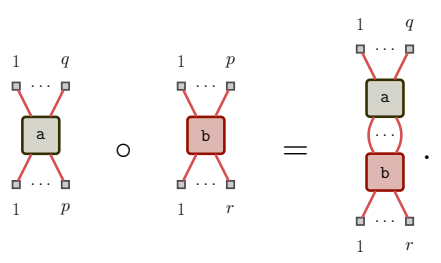

By definition, connecting the input (resp. output) of a wire to the output (resp. input) of a prograph $x$ does not change $x$.

The input (resp. output) arity of a prograph $x$ is its number of inputs i $(x)$ (resp. outputs o $(x)$ ). The inputs (resp. outputs) of a prograph are numbered from left to right from 1 to $\mathrm{i}(x)$ (resp. $\mathrm{o}(x)$ ), possibly implicitly in the drawings. The degree $\operatorname{deg}(x)$ of a prograph $x$ is the number of 
elementary prographs required to build it. For instance,

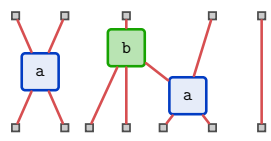

is a prograph over $G:=G(2,2) \sqcup G(3,1)$ where $G(2,2):=\{\mathrm{a}\}$ and $G(3,1):=\{\mathrm{b}\}$. Its input arity is 7 , its output arity is 5 , and its degree is 3 .

The free PRO generated by $G$ is the PRO Free $(G)$ whose elements are all the prographs on $G$, the horizontal composition being the operation $*$ on prographs, and the vertical composition being the operation $\circ$. Its unit $\mathbb{1}_{1}$ is the wire, and for any $p \geqslant 0, \mathbb{1}_{p}$ is the horizontal composition of $p$ occurrences of the wire. Notice that by (1.3.10), there is no elementary prograph in Free $(G)$ with a null input or output arity. Therefore, $\mathbb{1}_{0}$ is the only element of $\operatorname{Free}(G)$ with a null input (resp. output) arity. In this work, we consider only free PROs satisfying this property.

Lemma 1.1. Let $\mathcal{P}$ be a free $P R O$ and $x, y, z, t \in \mathcal{P}$ such that $x * y=z \circ t$. Then, there exist four unique elements $x_{1}, x_{2}, y_{1}, y_{2}$ of $\mathcal{P}$ such that $x=x_{1} \circ x_{2}, y=y_{1} \circ y_{2}, z=x_{1} * y_{1}$, and $t=x_{2} * y_{2}$.

Proof. Let us prove the uniqueness. Assume that there are $x_{1}, x_{2}, y_{1}, y_{2} \in \mathcal{P}$ and $x_{1}^{\prime}, x_{2}^{\prime}, y_{1}^{\prime}, y_{2}^{\prime} \in \mathcal{P}$ such that $x=x_{1} \circ x_{2}=x_{1}^{\prime} \circ x_{2}^{\prime}, y=y_{1} \circ y_{2}=y_{1}^{\prime} \circ y_{2}^{\prime}, z=x_{1} * y_{1}=x_{1}^{\prime} * y_{1}^{\prime}$, and $t=x_{2} * y_{2}=x_{2}^{\prime} * y_{2}^{\prime}$. Then, we have in particular $\mathrm{o}\left(x_{1}\right)=\mathrm{o}\left(x_{1}^{\prime}\right)$ and $\mathrm{o}\left(y_{1}\right)=\mathrm{o}\left(y_{1}^{\prime}\right)$. This, together with the relation $x_{1} * y_{1}=x_{1}^{\prime} * y_{1}^{\prime}$ and the fact that $\mathcal{P}$ is free, implies $x_{1}=x_{1}^{\prime}$ and $y_{1}=y_{1}^{\prime}$. In the same way, we obtain $x_{2}=x_{2}^{\prime}$ and $y_{2}=y_{2}^{\prime}$.

Let us now give a geometrical proof for the existence based upon the fact that prographs are planar objects. Since $u:=x * y=z \circ t, u$ is a prograph obtained by an horizontal composition of two prographs. Then, $u=x * y$ depicted in a plane $\mathfrak{P}$ can be split into two regions $\mathfrak{X}$ and $\mathfrak{Y}$ such that $\mathfrak{X}$ contains the prograph $x, \mathfrak{Y}$ contains the prograph $y$, and $y$ is at the right of $x$. On the other hand, $u=z \circ t$ depicted in the same plane $\mathfrak{P}$ can be split into two regions $\mathfrak{Z}$ and $\mathfrak{T}$ such that $\mathfrak{Z}$ contains the prograph $z, \mathfrak{T}$ contains the prograph $t$, and $t$ is below $z$, the inputs of $z$ being connected to the outputs of $t$. We then obtain a division of $\mathfrak{P}$ into four regions $\mathfrak{X} \cap \mathfrak{Z}$, $\mathfrak{X} \cap \mathfrak{T}, \mathfrak{Y} \cap \mathfrak{Z}$, and $\mathfrak{Y} \cap \mathfrak{T}$, respectively containing prographs $x_{1}, x_{2}, y_{1}$, and $y_{2}$, and such that $x_{1} * y_{1}=z, x_{2} * y_{2}=t, x_{1} \circ x_{2}=x$, and $y_{1} \circ y_{2}=y$.

1.3.3. Reduced and indecomposable elements. Let $\mathcal{P}$ be a free PRO. Since $\mathcal{P}$ is free, any element $x$ of $\mathcal{P}$ can be uniquely written as $x=x_{1} * \cdots * x_{\ell}$ where the $x_{i}$ are elements of $\mathcal{P}$ different from $\mathbb{1}_{0}$, and $\ell \geqslant 0$ is maximal. We call the word $\operatorname{dec}(x):=\left(x_{1}, \ldots, x_{\ell}\right)$ the maximal decomposition of $x$ and the $x_{i}$ the factors of $x$. Notice that the maximal decomposition of $\mathbb{1}_{0}$ is the empty word. We have, for instance,

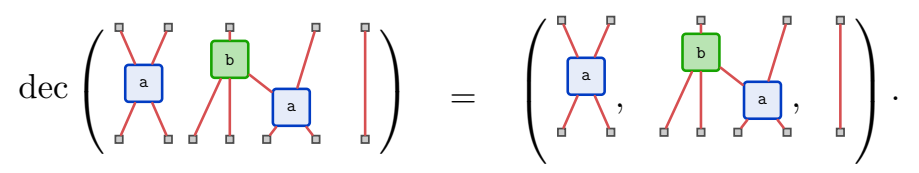


An element $x$ of $\mathcal{P}$ is reduced if all its factors are different from $\mathbb{1}_{1}$. For any element $x$ of $\mathcal{P}$, we denote by $\operatorname{red}(x)$ the reduced element of $\mathcal{P}$ admitting as maximal decomposition the longest subword of $\operatorname{dec}(x)$ consisting in factors different from $\mathbb{1}_{1}$. We have, for instance,

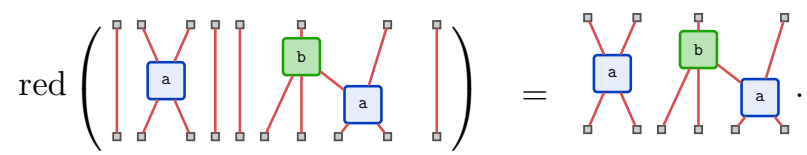

By extension, we denote by $\operatorname{red}(\mathcal{P})$ the set of the reduced elements of $\mathcal{P}$. Note that $\mathbb{1}_{0}$ belongs to $\operatorname{red}(\mathcal{P})$.

Besides, we say that an element $x$ of $\mathcal{P}$ is indecomposable if its maximal decomposition consists in exactly one factor. Note that $\mathbb{1}_{0}$ is not indecomposable while $\mathbb{1}_{1}$ is.

Lemma 1.2. Let $\mathcal{P}$ be a free $P R O$ and $x, y \in \mathcal{P}$, such that $x=\operatorname{red}(y)$. Then, by denoting by $\left(x_{1}, \ldots, x_{\ell}\right)$ the maximal decomposition of $x$, there exists a unique sequence of nonnegative integers $p_{1}, \ldots, p_{\ell}, p_{\ell+1}$ such that

$$
y=\mathbb{1}_{p_{1}} * x_{1} * \mathbb{1}_{p_{2}} * x_{2} * \cdots * x_{\ell} * \mathbb{1}_{p_{\ell+1}} .
$$

Proof. The existence comes from the fact that, since $x=\operatorname{red}(y)$, the maximal decomposition of $x$ is obtained from the one of $y$ by suppressing the factors equal to the wire. The uniqueness comes from the fact that $\mathcal{P}$ is a free monoid for the horizontal composition $*$.

\section{From PROs to combinatorial Hopf algebras}

We introduce in this section the main construction of this work and review some of its properties. In all this section, $\mathcal{P}$ is a free PRO generated by a bigraded set $G$. Starting with $\mathcal{P}$, our construction produces a bialgebra $\mathrm{H}(\mathcal{P})$ whose bases are indexed by the reduced elements of $\mathcal{P}$. We shall also extend this construction over a class of non necessarily free PROs.

2.1. The Hopf algebra of a free PRO. The bases of the vector space

$$
\mathrm{H}(\mathcal{P}):=\operatorname{Vect}(\operatorname{red}(\mathcal{P}))
$$

are indexed by the reduced elements of $\mathcal{P}$. The elements $\mathbf{S}_{x}, x \in \operatorname{red}(\mathcal{P})$, form thus a basis of $\mathrm{H}(\mathcal{P})$, called fundamental basis. We endow $\mathrm{H}(\mathcal{P})$ with a product $\cdot: \mathrm{H}(\mathcal{P}) \otimes \mathrm{H}(\mathcal{P}) \rightarrow \mathrm{H}(\mathcal{P})$ linearly defined, for any reduced elements $x$ and $y$ of $\mathcal{P}$, by

$$
\mathbf{S}_{x} \cdot \mathbf{S}_{y}:=\mathbf{S}_{x * y}
$$

and with a coproduct $\Delta: \mathrm{H}(\mathcal{P}) \rightarrow \mathrm{H}(\mathcal{P}) \otimes \mathrm{H}(\mathcal{P})$ linearly defined, for any reduced elements $x$ of $\mathcal{P}$, by

$$
\Delta\left(\mathbf{S}_{x}\right):=\sum_{\substack{y, z \in \mathcal{P} \\ y \circ z=x}} \mathbf{S}_{\operatorname{red}(y)} \otimes \mathbf{S}_{\operatorname{red}(z)}
$$


Throughout this section, we shall consider some examples involving the free PRO generated by $G:=G(2,2) \sqcup G(3,1)$ where $G(2,2):=\{\mathrm{a}\}$ and $G(3,1):=\{\mathrm{b}\}$, denoted by AB. For instance, we have in $\mathrm{H}(\mathrm{AB})$

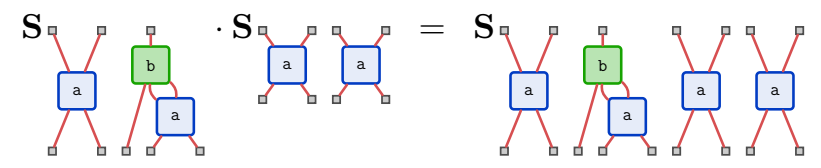

and

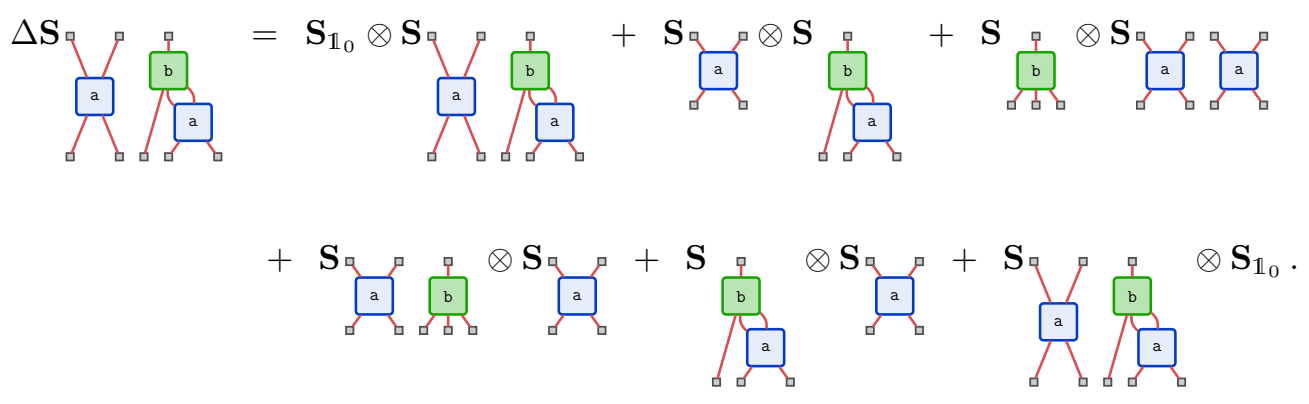

Lemma 2.1. Let $\mathcal{P}$ be a free $P R O$. Then, the coproduct $\Delta$ of $\mathrm{H}(\mathcal{P})$ is coassociative.

Proof. The bases of the vector space $\operatorname{Vect}(\mathcal{P})$ are indexed by the (non-necessarily reduced) elements of $\mathcal{P}$. Then, the elements $R_{x}, x \in \mathcal{P}$, form a basis of $\operatorname{Vect}(\mathcal{P})$. Let us consider the coproduct $\Delta^{\prime}$ defined, for any $x \in \mathcal{P}$, by

$$
\Delta^{\prime}\left(R_{x}\right):=\sum_{\substack{y, z \in \mathcal{P} \\ y \circ z=x}} R_{y} \otimes R_{z} .
$$

The associativity of the vertical composition $\circ$ of $\mathcal{P}$ (see (1.3.5)) implies that $\Delta^{\prime}$ is coassociative and hence, that $\operatorname{Vect}(\mathcal{P})$ together with $\Delta^{\prime}$ form a coalgebra.

Consider now the map $\phi: \operatorname{Vect}(\mathcal{P}) \rightarrow \mathrm{H}(\mathcal{P})$ defined, for any $x \in \mathcal{P}$, by $\phi\left(R_{x}\right):=\mathbf{S}_{\text {red }(x)}$. Let us show that $\phi$ commutes with the coproducts $\Delta$ and $\Delta^{\prime}$, that is, $(\phi \otimes \phi) \Delta^{\prime}=\Delta \phi$. Let $x \in \mathcal{P}$. By Lemma 1.2 , by denoting by $\left(x_{1}, \ldots, x_{\ell}\right)$ the maximal decomposition of $\operatorname{red}(x)$, there is a unique way to write $x$ as $x=\mathbb{1}_{p_{1}} * x_{1} * \cdots * x_{\ell} * \mathbb{1}_{\ell+1}$ where the $p_{i}$ are some integers. Then, thanks to 
the associativity of $*$ (see (1.3.4)), by iteratively applying Lemma 1.1, we have

$$
\begin{aligned}
(\phi \otimes \phi) \Delta^{\prime}\left(R_{x}\right)= & \sum_{\substack{y, z \in \mathcal{P} \\
y \circ z=x}} \mathbf{S}_{\operatorname{red}(y)} \otimes \mathbf{S}_{\operatorname{red}(z)} \sum_{\substack{y_{1}, \ldots, y_{\ell} \in \mathcal{P} \\
z_{1}, \ldots, z_{\ell} \in \mathcal{P} \\
\left(\mathbb{1}_{p_{1}} * y_{1} * \cdots * y_{\ell} * \mathbb{1}_{\ell+1}\right) \\
\circ\left(\mathbb{1}_{p_{1}} * z_{1} * \cdots * z_{\ell} * \mathbb{1}_{\ell+1}\right)=\\
\mathbb{1}_{p_{1}} * x_{1} * \cdots * x_{\ell} * \mathbb{1}_{\ell+1}}} \mathbf{S}_{\operatorname{red}\left(\mathbb{1}_{p_{1}} * y_{1} * \cdots * y_{\ell} * \mathbb{1}_{\ell+1}\right)} \otimes \mathbf{S}_{\operatorname{red}\left(\mathbb{1}_{p_{1}} * z_{1} * \cdots * z_{\ell} * \mathbb{1}_{\ell+1}\right)} \\
= & \sum_{\substack{y_{1}, \ldots, y_{\ell} \in \mathcal{P} \\
z_{1}, \ldots, z_{\ell} \in \mathcal{P}\\
}} \mathbf{S}_{\operatorname{red}\left(y_{1} * \cdots * y_{\ell}\right)} \otimes \mathbf{S}_{\operatorname{red}\left(z_{1} * \cdots * z_{\ell}\right)} \\
= & \sum_{\substack{y, z \in \mathcal{P} \\
y \circ z=\operatorname{red}(x)}} \mathbf{S}_{\operatorname{red}(y)} \otimes \mathbf{S}_{\operatorname{red}(z)} \\
= & \Delta\left(\phi\left(R_{x}\right)\right)
\end{aligned}
$$

Now, the coassociativity of $\Delta$ comes from the fact that $\phi$ is a surjective map commuting with $\Delta$ and $\Delta^{\prime}$. In more details, if $x$ is a reduced element of $\mathcal{P}$ and $I$ is the identity map on $\mathrm{H}(\mathcal{P})$, we have

$$
\begin{aligned}
(\Delta \otimes I) \Delta\left(\mathbf{S}_{x}\right) & =(\Delta \otimes I) \Delta\left(\phi\left(R_{x}\right)\right) \\
& =(\phi \otimes \phi \otimes \phi)\left(\Delta^{\prime} \otimes I\right) \Delta^{\prime}\left(R_{x}\right) \\
& =(\phi \otimes \phi \otimes \phi)\left(I \otimes \Delta^{\prime}\right) \Delta^{\prime}\left(R_{x}\right) \\
& =(I \otimes \Delta) \Delta\left(\phi\left(R_{x}\right)\right) \\
& =(I \otimes \Delta) \Delta\left(\mathbf{S}_{x}\right)
\end{aligned}
$$

Lemma 2.2. Let $\mathcal{P}$ be a free PRO. Then, the coproduct $\Delta$ of $\mathrm{H}(\mathcal{P})$ is a morphism of algebras.

Proof. Let $x$ and $y$ be two reduced elements of $\mathcal{P}$. We have

$$
\Delta\left(\mathbf{S}_{x} \cdot \mathbf{S}_{y}\right)=\sum_{\substack{z, t \in \mathcal{P} \\ z \circ t=x * y}} \mathbf{S}_{\operatorname{red}(z)} \otimes \mathbf{S}_{\mathrm{red}(t)}
$$

and

$$
\Delta\left(\mathbf{S}_{x}\right) \Delta\left(\mathbf{S}_{y}\right)=\sum_{\substack{x_{1}, x_{2}, y_{1}, y_{2} \in \mathcal{P} \\ x_{1} \circ x_{2}=x \\ y_{1} \circ y_{2}=y}} \mathbf{S}_{\operatorname{red}\left(x_{1}\right) * \operatorname{red}\left(y_{1}\right)} \otimes \mathbf{S}_{\operatorname{red}\left(x_{2}\right) * \operatorname{red}\left(y_{2}\right)}
$$

The coproduct $\Delta$ is a morphism of algebras if (2.1.17) and (2.1.18) are equal. Let us show that it is the case.

Assume that there are two elements $z$ and $t$ of $\mathcal{P}$ such that $z \circ t=x * y$. Then, the pair $(z, t)$ contributes to the coefficient of the tensor $\mathbf{S}_{\mathrm{red}(z)} \otimes \mathbf{S}_{\mathrm{red}(t)}$ in (2.1.17). Moreover, since $\mathcal{P}$ is free, by Lemma 1.1, there exist four unique elements $x_{1}, x_{2}, y_{1}$, and $y_{2}$ of $\mathcal{P}$ such that $x=x_{1} \circ x_{2}$, 
$y=y_{1} \circ y_{2}, z=x_{1} * y_{1}$, and $t=x_{2} * y_{2}$. Then, $\operatorname{since} \operatorname{red}\left(x_{1}\right) * \operatorname{red}\left(y_{1}\right)=\operatorname{red}\left(x_{1} * y_{1}\right)=\operatorname{red}(z)$ and $\operatorname{red}\left(x_{2}\right) * \operatorname{red}\left(y_{2}\right)=\operatorname{red}\left(x_{2} * y_{2}\right)=\operatorname{red}(t)$, the quadruple $\left(x_{1}, x_{2}, y_{1}, y_{2}\right)$, wholly and uniquely determined by the pair $(z, t)$, contributes to the coefficient of the tensor $\mathbf{S}_{\operatorname{red}(z)} \otimes \mathbf{S}_{\operatorname{red}(t)}$ in (2.1.18).

Conversely, assume that there are four elements $x_{1}, x_{2}, y_{1}$, and $y_{2}$ in $\mathcal{P}$ such that $x_{1} \circ x_{2}=x$ and $y_{1} \circ y_{2}=y$. Then, the quadruple $\left(x_{1}, x_{2}, y_{1}, y_{2}\right)$ contributes to the coefficient of the tensor $\mathbf{S}_{\mathrm{red}\left(x_{1}\right) * \operatorname{red}\left(y_{1}\right)} \otimes \mathbf{S}_{\mathrm{red}\left(x_{2}\right) * \operatorname{red}\left(y_{2}\right)}$ in (2.1.18). Now, by (1.3.6), we have

$$
x * y=\left(x_{1} \circ x_{2}\right) *\left(y_{1} \circ y_{2}\right)=\left(x_{1} * y_{1}\right) \circ\left(x_{2} * y_{2}\right) .
$$

Then, since $\operatorname{red}\left(x_{1}\right) * \operatorname{red}\left(y_{1}\right)=\operatorname{red}\left(x_{1} * y_{1}\right)$ and $\operatorname{red}\left(x_{2}\right) * \operatorname{red}\left(y_{2}\right)=\operatorname{red}\left(x_{2} * y_{2}\right)$, the pair $\left(x_{1} *\right.$ $\left.y_{1}, x_{2} * y_{2}\right)$, wholly and uniquely determined by the quadruple $\left(x_{1}, x_{2}, y_{1}, y_{2}\right)$, contributes to the coefficient of the tensor $\mathbf{S}_{\mathrm{red}\left(x_{1}\right) * \operatorname{red}\left(y_{1}\right)} \otimes \mathbf{S}_{\operatorname{red}\left(x_{2}\right) * \operatorname{red}\left(y_{2}\right)}$ in (2.1.17).

Hence, the coefficient of any tensor is the same in (2.1.17) and in (2.1.18). Then, these expressions are equal.

Theorem 2.3. Let $\mathcal{P}$ be a free $P R O$. Then, $\mathrm{H}(\mathcal{P})$ is a bialgebra.

Proof. The associativity of - comes directly from the associativity of the horizontal composition * of $\mathcal{P}$ (see (1.3.4)). Moreover, by Lemmas 2.1 and 2.2, the coproduct of $\mathbf{H}(\mathcal{P})$ is coassociative and is a morphism of algebras. Thus, $\mathrm{H}(\mathcal{P})$ is a bialgebra.

2.2. Properties of the construction. Let us now study the general properties of the bialgebras obtained by the construction $\mathrm{H}$.

\subsubsection{Algebraic generators and freeness.}

Proposition 2.4. Let $\mathcal{P}$ be a free PRO. Then, $\mathrm{H}(\mathcal{P})$ is freely generated as an algebra by the set of all $\mathbf{S}_{g}$, where the $g$ are indecomposable and reduced elements of $\mathcal{P}$.

Proof. Any reduced element $x$ of $\mathcal{P}$ can be written as $x=x_{1} * \cdots * x_{\ell}$ where $\left(x_{1}, \ldots, x_{\ell}\right)$ is the maximal decomposition of $x$. This implies that in $\mathrm{H}(\mathcal{P})$, we have $\mathbf{S}_{x}=\mathbf{S}_{x_{1}} \cdot \ldots \cdot \mathbf{S}_{x_{\ell}}$. Since for all $i \in[\ell]$, the $\mathbf{S}_{x_{i}}$ are indecomposable and reduced elements of $\mathcal{P}$, the set of all $\mathbf{S}_{g}$ generates $\mathbf{H}(\mathcal{P})$. The uniqueness of the maximal decomposition of $x$ implies that $\mathrm{H}(\mathcal{P})$ is free on the $\mathbf{S}_{g}$.

2.2.2. Gradings. There are several ways to define gradings for $\mathrm{H}(\mathcal{P})$ to turn it into a combinatorial Hopf algebra. For this purpose, we say that a map $\omega: \operatorname{red}(\mathcal{P}) \rightarrow \mathbb{N}$ is a grading of $\mathcal{P}$ if it satisfies the following four properties:

(G1) for any reduced elements $x$ and $y$ of $\mathcal{P}, \omega(x * y)=\omega(x)+\omega(y)$;

(G2) for any reduced elements $x$ of $\mathcal{P}$ satisfying $x=y \circ z$ where $y, z \in \mathcal{P}, \omega(x)=\omega(\operatorname{red}(y))+$ $\omega(\operatorname{red}(z))$

(G3) for any $n \geqslant 0$, the fiber $\omega^{-1}(n)$ is finite;

(G4) $\omega^{-1}(0)=\left\{\mathbb{1}_{0}\right\}$. 
A very generic way to endow $\mathcal{P}$ with a grading consists in providing a map $\omega: G \rightarrow \mathbb{N} \backslash\{0\}$ associating a positive integer with any generator of $\mathcal{P}$, namely its weight; the degree $\omega(x)$ of any element $x$ of $\mathcal{P}$ being the sum of the weights of the occurrences of the generators used to build $x$. For instance, the map $\omega$ defined by $\omega(\mathrm{a}):=3$ and $\omega(\mathrm{b}):=2$ is a grading of $A B$ and we have

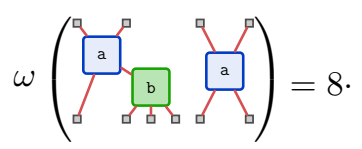

Proposition 2.5. Let $\mathcal{P}$ be a free $P R O$ and $\omega$ be a grading of $\mathcal{P}$. Then, with the grading

$$
\mathrm{H}(\mathcal{P})=\bigoplus_{n \geqslant 0} \operatorname{Vect}\left(\mathbf{S}_{x}: x \in \operatorname{red}(\mathcal{P}) \text { and } \omega(x)=n\right),
$$

$\mathrm{H}(\mathcal{P})$ is a combinatorial Hopf algebra.

Proof. Notice first that $\mathbf{S}_{\mathbb{1}_{0}}$ is the neutral element of the product of $\mathbf{H}(\mathcal{P})$, and, for any reduced element $x$ of $\mathcal{P}$ of degree different from 0 , the coproduct $\Delta\left(\mathbf{S}_{x}\right)$ contains the tensors $\mathbf{S}_{\mathbb{1}_{0}} \otimes \mathbf{S}_{x}$ and $\mathbf{S}_{x} \otimes \mathbf{S}_{\mathbb{1}_{0}}$. Then, together with the fact that by (G4), $\mathbf{S}_{\mathbb{1}_{0}}$ is an element of the homogeneous component of degree 0 of $\mathbf{H}(\mathcal{P}), \mathbf{H}(\mathcal{P})$ admits a unit and a counit. Moreover, (G3) implies that for all $n \geqslant 0$, the homogeneous components of degree $n$ of $\mathrm{H}(\mathcal{P})$ are finite, and $\left(G_{4}\right)$ implies that $\mathrm{H}(\mathcal{P})$ is connected.

Besides, respectively by (G1) and by (G2), the grading provided by $\omega$ is compatible with the product and the coproduct of $\mathrm{H}(\mathcal{P})$.

Hence, together with the fact that, by Theorem $2.3, \mathrm{H}(\mathcal{P})$ is a bialgebra, it is also a combinatorial Hopf algebra.

2.2.3. Antipode. Since the antipode of a combinatorial Hopf algebra can be computed by induction on the degree, we obtain an expression for the one of $\mathrm{H}(\mathcal{P})$ when $\mathcal{P}$ admits a grading. This expression is an instance of the Takeuchi formula [Tak71] and is particularly simple since the product of $\mathrm{H}(\mathcal{P})$ is multiplicative.

Proposition 2.6. Let $\mathcal{P}$ be a free PRO admitting a grading. For any reduced element $x$ of $\mathcal{P}$ different from $\mathbb{1}_{0}$, the antipode $\nu$ of $\mathrm{H}(\mathcal{P})$ satisfies

$$
\nu\left(\mathbf{S}_{x}\right)=\sum_{\substack{x_{1}, \ldots, x_{\ell} \in \mathcal{P}, \ell \geqslant 1 \\ x_{1} 0.0 x_{\ell}=x \\ \operatorname{red}\left(x_{i}\right) \neq \mathbb{1}_{0}, i \in[\ell]}}(-1)^{\ell} \mathbf{S}_{\operatorname{red}\left(x_{1} * \cdots * x_{\ell}\right)} .
$$

Proof. By Proposition 2.5, $\mathrm{H}(\mathcal{P})$ is a combinatorial Hopf algebra, and hence, the antipode $\nu$, which is the inverse of the identity morphism for the convolution product, exists and is unique. Then, for any reduced element $x$ of $\mathcal{P}$ different from $\mathbb{1}_{0}$,

$$
\nu\left(\mathbf{S}_{x}\right)=-\mathbf{S}_{x}-\sum_{\substack{y, z \in \mathcal{P} \backslash\{x\} \\ y \circ z=x}} \nu\left(\mathbf{S}_{\operatorname{red}(y)}\right) \cdot \mathbf{S}_{\operatorname{red}(z)} .
$$

Expression (2.2.3) for $\nu$ follows now by induction on the degree of $x$ in $\mathcal{P}$. 
We have for instance in $\mathrm{H}(\mathrm{AB})$,

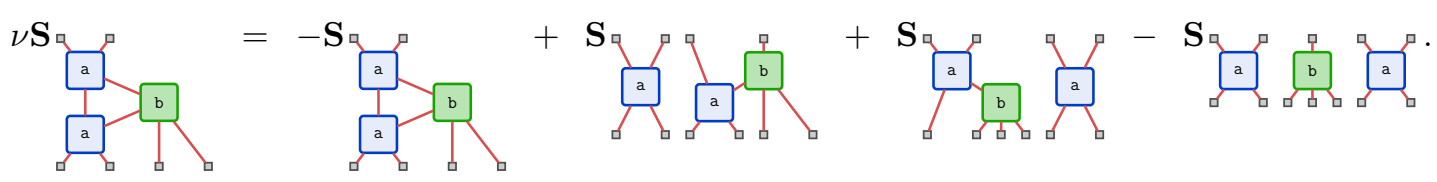

2.2.4. Duality. When $\mathcal{P}$ admits a grading, let us denote by $\mathrm{H}(\mathcal{P})^{\star}$ the graded dual of $\mathrm{H}(\mathcal{P})$. By definition, the adjoint basis of the fundamental basis of $\mathrm{H}(\mathcal{P})$ consists in the elements $\mathbf{S}_{x}^{\star}$, $x \in \operatorname{red}(\mathcal{P})$.

Proposition 2.7. Let $\mathcal{P}$ be a free PRO admitting a grading. Then, for any reduced elements $x$ and $y$ of $\mathcal{P}$, the product and the coproduct of $\mathrm{H}(\mathcal{P})^{\star}$ satisfy

$$
\mathbf{S}_{x}^{\star} \cdot \mathbf{S}_{y}^{\star}=\sum_{\begin{array}{c}
x^{\prime}, y^{\prime} \in \mathcal{P} \\
x^{\prime} \circ y^{\prime} \in \operatorname{red}(\mathcal{P}) \\
\operatorname{red}\left(x^{\prime}\right)=x, \operatorname{red}\left(y^{\prime}\right)=y
\end{array}} \mathbf{S}_{x^{\prime} \circ y^{\prime}}^{\star}
$$

and

$$
\Delta\left(\mathbf{S}_{x}^{\star}\right)=\sum_{\substack{y, z \in \mathcal{P} \\ y * z=x}} \mathbf{S}_{y}^{\star} \otimes \mathbf{S}_{z}^{\star}
$$

Proof. Let us denote by $\langle-,-\rangle: \mathrm{H}(\mathcal{P}) \otimes \mathrm{H}(\mathcal{P})^{\star} \rightarrow \mathbb{C}$ the duality bracket between $\mathrm{H}(\mathcal{P})$ and its graded dual.

By duality, we have

$$
\mathbf{S}_{x}^{\star} \cdot \mathbf{S}_{y}^{\star}=\sum_{z \in \operatorname{red}(\mathcal{P})}\left\langle\Delta\left(\mathbf{S}_{z}\right), \mathbf{S}_{x}^{\star} \otimes \mathbf{S}_{y}^{\star}\right\rangle \mathbf{S}_{z}^{\star}
$$

Expression (2.2.6) follows from the fact that for any reduced element $z$ of $\mathcal{P}, \mathbf{S}_{x} \otimes \mathbf{S}_{y}$ appears in $\Delta\left(\mathbf{S}_{z}\right)$ if and only if there exist $x^{\prime}, y^{\prime} \in \mathcal{P}$ such that $x^{\prime} \circ y^{\prime}=z, \operatorname{red}\left(x^{\prime}\right)=x$ and $\operatorname{red}\left(y^{\prime}\right)=y$.

Besides, again by duality, we have

$$
\Delta\left(\mathbf{S}_{x}^{\star}\right)=\sum_{y, z \in \operatorname{red}(\mathcal{P})}\left\langle\mathbf{S}_{y} \cdot \mathbf{S}_{z}, \mathbf{S}_{x}^{\star}\right\rangle \mathbf{S}_{y}^{\star} \otimes \mathbf{S}_{z}^{\star} .
$$

Expression (2.2.7) follows from the fact that for any reduced elements $y$ and $z$ of $\mathcal{P}, \mathbf{S}_{x}$ appears in $\mathbf{S}_{y} \cdot \mathbf{S}_{z}$ if and only if $y * z=x$. 
For instance, we have in $\mathrm{H}(\mathrm{AB})$

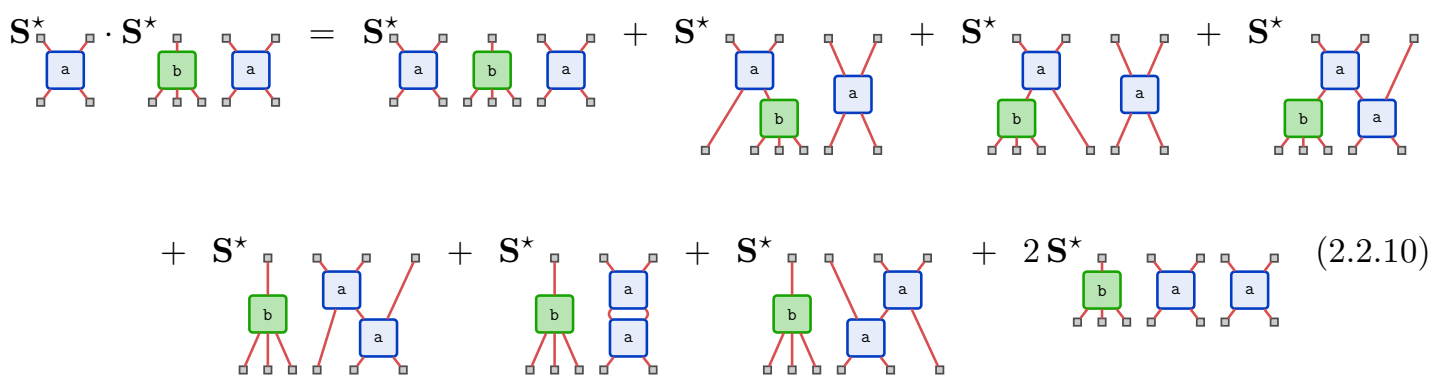

and

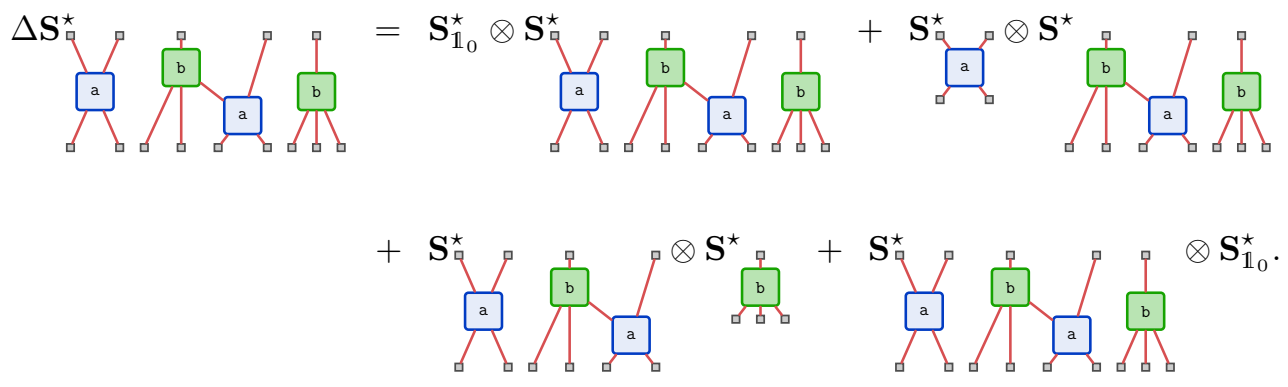

\subsubsection{Quotient bialgebras.}

Proposition 2.8. Let $G$ and $G^{\prime}$ be two bigraded sets such that $G^{\prime} \subseteq G$. Then, the map $\phi$ : $\mathrm{H}(\operatorname{Free}(G)) \rightarrow \mathrm{H}\left(\right.$ Free $\left.\left(G^{\prime}\right)\right)$ linearly defined, for any reduced element $x$ of $\operatorname{Free}(G)$, by

$$
\phi\left(\mathbf{S}_{x}\right):= \begin{cases}\mathbf{S}_{x} & \text { if } x \in \operatorname{Free}\left(G^{\prime}\right), \\ 0 & \text { otherwise, }\end{cases}
$$

is a surjective bialgebra morphism. Moreover, $\mathrm{H}\left(\operatorname{Free}\left(G^{\prime}\right)\right)$ is a quotient bialgebra of $\mathrm{H}(\operatorname{Free}(G))$.

Proof. Let $V$ be the linear span of the $\mathbf{S}_{x}$ where the $x$ are reduced elements of Free $(G) \backslash \operatorname{Free}\left(G^{\prime}\right)$. Immediately from the definitions of the product and the coproduct of $\mathrm{H}($ Free $(G))$, we observe that $V$ is a bialgebra ideal of $\mathrm{H}(\operatorname{Free}(G))$. The map $\phi$ is the canonical projection from $\mathrm{H}(\operatorname{Free}(G))$ to $\mathrm{H}(\operatorname{Free}(G)) / V \simeq \mathrm{H}\left(\operatorname{Free}\left(G^{\prime}\right)\right)$, whence the result.

2.3. The Hopf algebra of a stiff PRO. We now extend the construction $\mathrm{H}$ to a class a nonnecessarily free PROs. Still in this section, $\mathcal{P}$ is a free PRO.

Let $\equiv$ be a congruence of $\mathcal{P}$. For any element $x$ of $\mathcal{P}$, we denote by $[x]_{\equiv}$ (or by $[x]$ if the context is clear) the $\equiv$-equivalence class of $x$. We say that $\equiv$ is a stiff congruence if the following three properties are satisfied:

(C1) for any reduced element $x$ of $\mathcal{P}$, the set $[x]$ is finite;

(C2) for any reduced element $x$ of $\mathcal{P},[x]$ contains reduced elements only; 
(C3) for any two elements $x$ and $x^{\prime}$ of $\mathcal{P}$ such that $x \equiv x^{\prime}$, the maximal decompositions of $x$ and $x^{\prime}$ are, respectively of the form $\left(x_{1}, \ldots, x_{\ell}\right)$ and $\left(x_{1}^{\prime}, \ldots, x_{\ell}^{\prime}\right)$ for some $\ell \geqslant 0$, and for any $i \in[\ell], x_{i} \equiv x_{i}^{\prime}$.

We say that a PRO is a stiff PRO if it is the quotient of a free PRO by a stiff congruence.

For any $\equiv$-equivalence class $[x]$ of reduced elements of $\mathcal{P}$, set

$$
\mathbf{T}_{[x]}:=\sum_{x^{\prime} \in[x]} \mathbf{S}_{x^{\prime}} .
$$

Notice that thanks to $(C 1)$ and $(C 2), \mathbf{T}_{[x]}$ is a well-defined element of $\mathbf{H}(\mathcal{P})$.

For instance, if $\mathcal{P}$ is the quotient of the free PRO generated by $G:=G(1,1) \sqcup G(2,2)$ where $G(1,1):=\{\mathrm{a}\}$ and $G(2,2):=\{\mathrm{b}\}$ by the finest congruence $\equiv$ satisfying

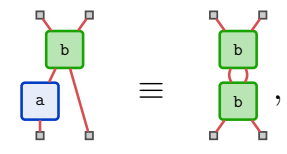

one has

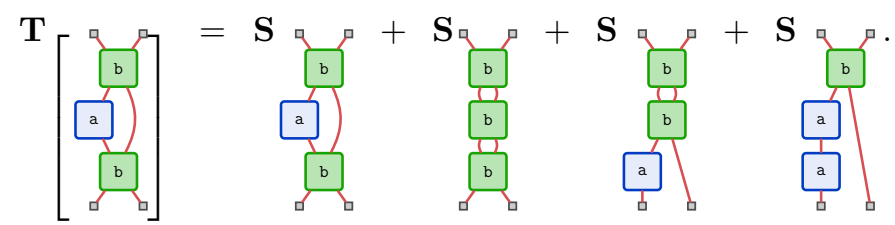

Moreover, we can observe that $\equiv$ is a stiff congruence.

If $\equiv$ is a stiff congruence of $\mathcal{P},(C 2)$ and (C3) imply that all the elements of a same $\equiv$ equivalence class $[x]$ have the same number of factors and are all reduced or all nonreduced. Then, by extension, we shall say that a $\equiv$-equivalence class $[x]$ of $\mathcal{P} / \equiv$ is indecomposable (resp. reduced) if all its elements are indecomposable (resp. reduced) in $\mathcal{P}$. In the same way, the wire of $\mathcal{P} / \equiv$ is the $\equiv$-equivalence class of the wire of $\mathcal{P}$.

We shall now study how the product and the coproduct of $\mathbf{H}(\mathcal{P})$ behave on the $\mathbf{T}_{[x]}$.

2.3.1. Product. Let us show that the linear span of the $\mathbf{T}_{[x]}$, where the $[x]$ are $\equiv$-equivalence classes of reduced elements of $\mathcal{P}$, forms a subalgebra of $\mathbf{H}(\mathcal{P})$. The product on the $\mathbf{T}_{[x]}$ is multiplicative and admits the following simple description.

Proposition 2.9. Let $\mathcal{P}$ be a free PRO and $\equiv$ be a stiff congruence of $\mathcal{P}$. Then, for any $\equiv-$ equivalence classes $[x]$ and $[y]$,

$$
\mathbf{T}_{[x]} \cdot \mathbf{T}_{[y]}=\mathbf{T}_{[x * y]},
$$

where $x$ (resp. $y$ ) is any element of $[x]$ (resp. $[y]$ ).

Proof. We have

$$
\mathbf{T}_{[x]} \cdot \mathbf{T}_{[y]}=\sum_{\substack{x^{\prime} \in[x] \\ y^{\prime} \in[y]}} \mathbf{S}_{x^{\prime} * y^{\prime}}
$$


and

$$
\mathbf{T}_{[x * y]}=\sum_{z \in[x * y]} \mathbf{S}_{z}
$$

Let us show that (2.3.5) and (2.3.6) are equal. It is enough to check that these sums have the same support. Indeed, (2.3.6) is by definition multiplicity free and (2.3.5) is multiplicity free because $\mathcal{P}$ is free, and all elements of a $\equiv$-equivalence class have the same input arity and the same output arity.

Assume that there is a reduced element $t$ of $\mathcal{P}$ such that $\mathbf{S}_{t}$ appears in (2.3.5). Then, one has $t=x^{\prime} * y^{\prime}$ for two reduced elements $x^{\prime}$ and $y^{\prime}$ of $\mathcal{P}$ such that $x^{\prime} \in[x]$ and $y^{\prime} \in[y]$. Since $\equiv$ is a congruence of PROs, we have $\left[x^{\prime} * y^{\prime}\right]=[x * y]$ and thus, $t \in[x * y]$. This shows that $\mathbf{S}_{t}$ also appears in (2.3.6).

Conversely, assume that there is a reduced element $z$ of $\mathcal{P}$ such that $\mathbf{S}_{z}$ appears in (2.3.6). Then, one has $z \in[x * y]$. Since $\equiv$ satisfies $(C 3)$, the maximal decomposition of $z$ satisfies $\operatorname{dec}(z)=\left(x_{1}^{\prime}, \ldots, x_{k}^{\prime}, y_{1}^{\prime}, \ldots, y_{\ell}^{\prime}\right)$ where $\operatorname{dec}(x)=\left(x_{1}, \ldots, x_{k}\right), \operatorname{dec}(y)=\left(y_{1}, \ldots, y_{\ell}\right), x_{i}^{\prime} \equiv x_{i}$, and $y_{j}^{\prime} \equiv y_{j}$ for all $i \in[k]$ and $j \in[\ell]$. Moreover, as $\equiv$ is a congruence of PROs, $x^{\prime}:=x_{1}^{\prime} * \cdots * x_{k}^{\prime} \equiv x$ and $y^{\prime}:=y_{1}^{\prime} * \cdots * y_{\ell}^{\prime} \equiv y$. We then have $z=x^{\prime} * y^{\prime}$ with $x^{\prime} \in[x]$ and $y^{\prime} \in[y]$. This shows that $\mathbf{S}_{z}$ also appears in (2.3.5).

2.3.2. Coproduct. To prove that the linear span of the $\mathbf{T}_{[x]}$, where the $[x]$ are $\equiv$-equivalence classes of reduced elements of $\mathcal{P}$, forms a subcoalgebra of $\mathrm{H}(\mathcal{P})$ and provides the description of the coproduct of a $\mathbf{T}_{[x]}$, we need the following notation. For any element $x$ of $\mathcal{P}$,

$$
\operatorname{red}([x]):=\left\{\operatorname{red}\left(x^{\prime}\right): x^{\prime} \in[x]\right\} .
$$

Lemma 2.10. Let $\mathcal{P}$ be a free $P R O$ and $\equiv$ be a stiff congruence of $\mathcal{P}$. For any element $x$ of $\mathcal{P}$,

$$
\operatorname{red}([x])=[\operatorname{red}(x)] \text {. }
$$

Proof. Let us denote by $y$ the element $\operatorname{red}(x)$ and let $y^{\prime} \in \operatorname{red}([x])$. Let us show that $y^{\prime} \in[\operatorname{red}(x)]$. By Definition (2.3.7), there is an element $x^{\prime}$ of $\mathcal{P}$ such that $x^{\prime} \in[x]$ and $\operatorname{red}\left(x^{\prime}\right)=y^{\prime}$. Since $\equiv$ satisfies $(C 3), \operatorname{dec}\left(x^{\prime}\right)$ and $\operatorname{dec}(x)$ have the same length $\ell$ and $\operatorname{dec}\left(x^{\prime}\right)_{i} \equiv \operatorname{dec}(x)_{i}$ for all $i \in[\ell]$. Moreover, since $\equiv$ satisfies $(C 2)$, for all $i \in[\ell] \operatorname{dec}\left(x^{\prime}\right)_{i}$ and $\operatorname{dec}(x)_{i}$ are both reduced elements or are both wires. Hence, since $\operatorname{dec}\left(y^{\prime}\right)$ and $\operatorname{dec}(y)$ are, respectively subwords of $\operatorname{dec}\left(x^{\prime}\right)$ and $\operatorname{dec}(x)$, they have the same length $k$ and $\operatorname{dec}\left(y^{\prime}\right)_{j} \equiv \operatorname{dec}(y)_{j}$ for all $j \in[k]$. Finally, since $\equiv$ is a congruence of PROs, $y^{\prime} \equiv y$. This shows that $y^{\prime} \in[\operatorname{red}(x)]$ and hence, $\operatorname{red}([x]) \subseteq[\operatorname{red}(x)]$.

Again, let us denote by $y$ the element $\operatorname{red}(x)$ and let $y^{\prime} \in[\operatorname{red}(x)]$. Let us show that $y^{\prime} \in \operatorname{red}([x])$. Since $y^{\prime} \equiv y$ and $\equiv$ satisfies $(C 3), \operatorname{dec}\left(y^{\prime}\right)$ and $\operatorname{dec}(y)$ have the same length $\ell$ and $\operatorname{dec}\left(y^{\prime}\right)_{i} \equiv \operatorname{dec}(y)_{i}$ for all $i \in[\ell]$. Moreover, since $y=\operatorname{red}(x)$, by Lemma 1.2, for some $p_{1}, \ldots, p_{\ell+1} \geqslant 0$, we have $x=\mathbb{1}_{p_{1}} * \operatorname{dec}(y)_{1} * \cdots * \mathbb{1}_{p_{\ell}} * \operatorname{dec}(y)_{\ell} * \mathbb{1}_{p_{\ell+1}}$. Now, by setting $x^{\prime}:=\mathbb{1}_{p_{1}} * \operatorname{dec}\left(y^{\prime}\right)_{1} * \cdots * \mathbb{1}_{p_{\ell}} * \operatorname{dec}\left(y^{\prime}\right)_{\ell} * \mathbb{1}_{p_{\ell+1}}$, the fact that $\equiv$ is a congruence of PROs implies $x^{\prime} \equiv x$. Since $y^{\prime}=\operatorname{red}\left(x^{\prime}\right)$, this shows that $y^{\prime} \in \operatorname{red}([x])$ and hence, $[\operatorname{red}(x)] \subseteq \operatorname{red}([x])$. 
Lemma 2.11. Let $\mathcal{P}$ be a free $P R O, \equiv$ be a stiff congruence of $\mathcal{P}$, and $y$ and $z$ be two elements of $\mathcal{P}$ such that $y \equiv z$. Then, $\operatorname{red}(y)=\operatorname{red}(z)$ implies $y=z$.

Proof. By contraposition, assume that $y \neq z$. Since $y \equiv z$ and $\equiv \operatorname{satisfies}(C 3), \operatorname{dec}(y)$ and $\operatorname{dec}(z)$ have the same length $\ell$, and $\operatorname{dec}(y)_{i} \equiv \operatorname{dec}(z)_{i}$ for all $i \in[\ell]$. Moreover, as $y \neq z$, there exists a $j \in[\ell]$ such that $\operatorname{dec}(y)_{j} \neq \operatorname{dec}(z)_{j}$. Now, since $\equiv \operatorname{satisfies}(C 2), \operatorname{dec}(y)_{j}$ and $\operatorname{dec}(z)_{j}$ are both reduced elements. Moreover, for all $i \in[\ell], \operatorname{dec}(y)_{i}$ and $\operatorname{dec}(z)_{i}$ are both reduced elements or are both wires. Hence, there is $j^{\prime} \geqslant 1$ such that $\operatorname{dec}(\operatorname{red}(y))_{j^{\prime}}=\operatorname{dec}(y)_{j}$ and $\operatorname{dec}(\operatorname{red}(z))_{j^{\prime}}=\operatorname{dec}(z)_{j}$. Since $\mathcal{P}$ is free, this implies that $\operatorname{red}(y) \neq \operatorname{red}(z)$.

Proposition 2.12. Let $\mathcal{P}$ be a free $P R O$ and $\equiv$ be a stiff congruence of $\mathcal{P}$. Then, for any $\equiv$-equivalence class $[x]$,

$$
\Delta\left(\mathbf{T}_{[x]}\right)=\sum_{\substack{[y],[z] \in \mathcal{P} / \equiv \\[y] \circ[z]=[x]}} \mathbf{T}_{\operatorname{red}([y])} \otimes \mathbf{T}_{\operatorname{red}([z])}
$$

Proof. We have

$$
\begin{aligned}
\Delta\left(\mathbf{T}_{[x]}\right) & =\sum_{\substack{y, z \in \mathcal{P} \\
y \circ z \in[x]}} \mathbf{S}_{\operatorname{red}(y)} \otimes \mathbf{S}_{\operatorname{red}(z)} \\
& =\sum_{\substack{y, z \in \mathcal{P} \\
[y] \circ[z]=[x]}} \mathbf{S}_{\operatorname{red}(y)} \otimes \mathbf{S}_{\operatorname{red}(z)} \\
& =\sum_{\substack{[y],[z] \in \mathcal{P} / \equiv \\
[y] \circ[z]=[x]}} \sum_{\substack{y^{\prime} \in[y] \\
z^{\prime} \in[z]}} \mathbf{S}_{\operatorname{red}\left(y^{\prime}\right)} \otimes \mathbf{S}_{\operatorname{red}\left(z^{\prime}\right)} \\
& =\sum_{\substack{[y],[z] \in \mathcal{P} / \equiv \\
[y] \circ[z]=[x]}} \sum_{y^{\prime} \in \operatorname{red}([y])} \mathbf{S}_{y^{\prime}} \otimes \mathbf{S}_{z^{\prime}} \\
& =\sum_{\substack{[y],[z] \in \mathcal{P} / \equiv \\
[y] \circ[z]=[x]}} \mathbf{T}_{\operatorname{red}([y])} \otimes \mathbf{T}_{\operatorname{red}([z])} .
\end{aligned}
$$

Let us comment the non-obvious equalities appearing in this computation. The equality between (2.3.10) and (2.3.11) comes from the fact that $\equiv$ is a congruence of PROs. The equality between (2.3.12) and (2.3.13) is a consequence of Lemma 2.11. Finally, (2.3.13) is, thanks to Lemma 2.10, equal to (2.3.14).

2.3.3. Sub-bialgebra. The description of the product and the coproduct on the $\mathbf{T}_{[x]}$ leads to the following result.

Theorem 2.13. Let $\mathcal{P}$ be a free PRO and $\equiv$ be a stiff congruence of $\mathcal{P}$. Then, the linear span of the $\mathbf{T}_{[x]}$, where the $[x]$ are $\equiv$-equivalence classes of reduced elements of $\mathcal{P}$, forms a sub-bialgebra of $\mathrm{H}(\mathcal{P})$. 
Proof. By Propositions 2.9 and 2.12, the product and the coproduct of $\mathrm{H}(\mathcal{P})$ are still well-defined on the $\mathbf{T}_{[x]}$. Then, since the $\mathbf{T}_{[x]}$ are by (2.3.1) sums of some $\mathbf{S}_{x^{\prime}}$, this implies the statement of the theorem.

We shall denote, by a slight abuse of notation, by $\mathrm{H}(\mathcal{P} / \equiv)$ the sub-bialgebra of $\mathrm{H}(\mathcal{P})$ spanned by the $\mathbf{T}_{[x]}$, where the $[x]$ are $\equiv$-equivalence classes of reduced elements of $\mathcal{P}$. Notice that the construction $\mathrm{H}$ as it was presented in Section 2.1 is a special case of this latter when $\equiv$ is the most refined congruence of PROs.

Note that this construction of sub-bialgebras of $\mathrm{H}(\mathcal{P})$ by taking an equivalence relation satisfying some precise properties and by considering the elements obtained by summing over its equivalence classes is analog to the construction of certain sub-bialgebras of the MalvenutoReutenauer Hopf algebra [MR95]. Indeed, some famous Hopf algebras are obtained in this way, as the Loday-Ronco Hopf algebra [LR98] by using the sylvester monoid congruence [HNT05], or the Poirier-Reutenauer Hopf algebra [PR95] by using the plactic monoid congruence [DHT02,HNT05].

2.3.4. The importance of the stiff congruence condition. Let us now explain why the stiff congruence condition required as a premise of Theorem 2.13 is important by providing an example of a non-stiff congruence of PROs failing to produce a bialgebra.

Consider the PRO $\mathcal{P}$ quotient of the free PRO generated by $G:=G(1,1) \sqcup G(2,2)$ where $G(1,1):=\{\mathrm{a}\}$ and $G(2,2):=\{\mathrm{b}\}$ by the finest congruence $\equiv$ satisfying

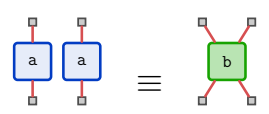

Here, $\equiv$ is not a stiff congruence since it satisfies (C2) but not (C3).

We have

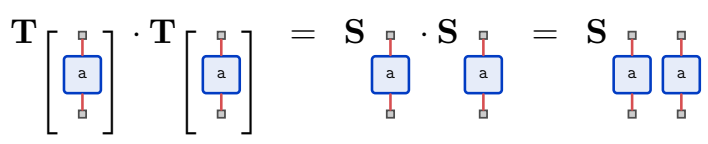

but this last element cannot be expressed on the $\mathbf{T}_{[x]}$. 
Besides, by a straightforward computation, we have

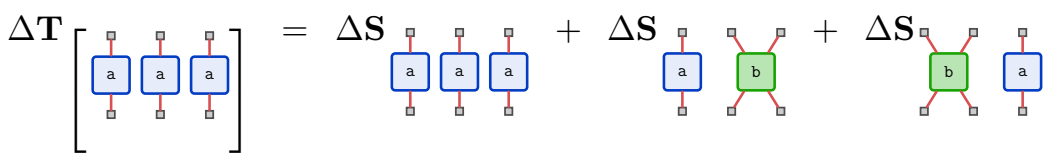

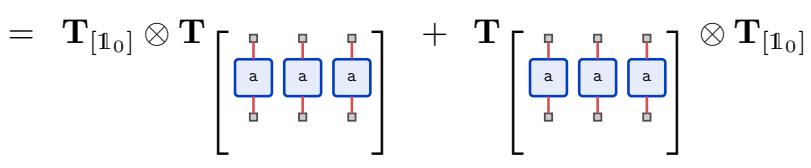

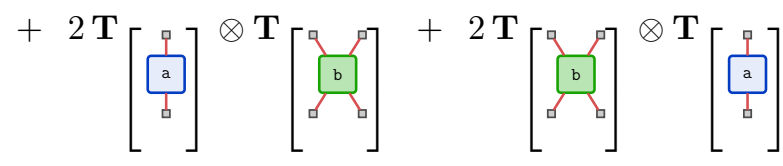

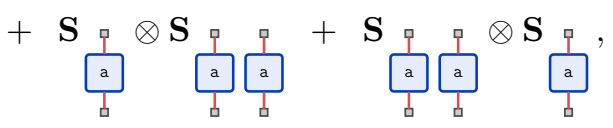

showing that the coproduct is neither well-defined on the $\mathbf{T}_{[x]}$.

2.3.5. Properties. By using similar arguments as those used to establish Proposition 2.4 together with the fact that $\equiv$ satisfies (C3) and the product formula of Proposition 2.9, we obtain that $\mathrm{H}(\mathcal{P} / \equiv)$ is freely generated as an algebra by the $\mathbf{T}_{[x]}$ where the $[x]$ are $\equiv$-equivalence classes of indecomposable and reduced elements of $\mathcal{P}$. Moreover, when $\omega$ is a grading of $\mathcal{P}$ so that all elements of a same $\equiv$-equivalence class have the same degree, the bialgebra $\mathrm{H}(\mathcal{P} / \equiv)$ is graded by the grading inherited from the one of $H(\mathcal{P})$ and forms hence a combinatorial Hopf algebra.

Proposition 2.14. Let $\mathcal{P}$ be a free $P R O$ and $\equiv_{1}$ and $\equiv_{2}$ be two stiff congruences of $\mathcal{P}$ such that $\equiv_{1}$ is finer than $\equiv_{2}$. Then, $\mathrm{H}\left(\mathcal{P} / \equiv_{2}\right)$ is a sub-bialgebra of $\mathrm{H}\left(\mathcal{P} / \equiv_{1}\right)$.

Proof. Since $\equiv_{1}$ is finer that $\equiv_{2}$, any $\mathbf{T}_{[x]_{\equiv_{2}}}$, where $[x]_{\equiv_{2}}$ is a $\equiv_{2}$-equivalence class of reduced elements of $\mathcal{P}$, is a sum of some $\mathbf{T}_{\left[x^{\prime}\right]_{\equiv_{1}}}$. More precisely, we have

$$
\mathbf{T}_{[x]_{\equiv_{2}}}=\sum_{\left[x^{\prime}\right] \equiv_{1} \subseteq[x]_{\equiv_{2}}} \mathbf{T}_{\left[x^{\prime}\right]_{\equiv_{1}}},
$$

implying the result.

2.4. Related constructions. In this section, we first describe two constructions allowing to build stiff PROs. The main interest of these constructions is that the obtained stiff PROs can be placed at the input of the construction $\mathrm{H}$. We next present a way to recover the natural Hopf algebra of an operad through the construction $\mathrm{H}$ and the previous constructions of stiff PROs. 
2.4.1. From operads to stiff PROs. Any operad $\mathcal{O}$ gives naturally rise to a $\operatorname{PRO} \mathrm{R}(\mathcal{O})$ whose elements are sequences of elements of $\mathcal{O}$ (see [Mar08]).

We recall here this construction. Let us set $\mathrm{R}(\mathcal{O}):=\sqcup_{p \geqslant 0} \sqcup_{q \geqslant 0} \mathrm{R}(\mathcal{O})(p, q)$ where

$$
\mathrm{R}(\mathcal{O})(p, q):=\left\{x_{1} \ldots x_{q}: x_{i} \in \mathcal{O}\left(p_{i}\right) \text { for all } i \in[q] \text { and } p_{1}+\cdots+p_{q}=p\right\} .
$$

The horizontal composition of $\mathrm{R}(\mathcal{O})$ is the concatenation of sequences, and the vertical composition of $\mathrm{R}(\mathcal{O})$ comes directly from the composition map of $\mathcal{O}$. More precisely, for any $x_{1} \ldots x_{r} \in \mathrm{R}(\mathcal{O})(q, r)$ and $y_{11} \ldots y_{1 q_{1}} \ldots y_{r 1} \ldots y_{r q_{r}} \in \mathrm{R}(\mathcal{O})(p, q)$, we have

$$
x_{1} \ldots x_{r} \circ y_{11} \ldots y_{1 q_{1}} \ldots y_{r 1} \ldots y_{r q_{r}}:=x_{1} \circ\left[y_{11}, \ldots, y_{1 q_{1}}\right] \ldots x_{r} \circ\left[y_{r 1}, \ldots, y_{r q_{r}}\right],
$$

where for any $i \in[r], x_{i} \in \mathcal{O}\left(q_{i}\right)$ and the occurrences of $\circ$ in the right-member of (2.4.2) refer to the total composition map of $\mathcal{O}$.

For instance, if $\mathcal{O}$ is the free operad generated by a generator of arity $2, \mathcal{O}$ is an operad involving binary trees. Then, the elements of the $\mathrm{PRO} R(\mathcal{O})$ are forests of binary trees. The horizontal composition of $\mathrm{R}(\mathcal{O})$ is the concatenation of forests, and the vertical composition $F_{1} \circ F_{2}$ in $\mathrm{R}(\mathcal{O})$, defined only between two forests $F_{1}$ and $F_{2}$ such that the number of leaves of $F_{1}$ is the same as the number of trees in $F_{2}$, consists in the forest obtained by grafting, from left to right, the roots of the trees of $F_{2}$ on the leaves of $F_{1}$.

Proposition 2.15. Let $\mathcal{O}$ be an operad such that the monoid $\left(\mathcal{O}(1), \circ_{1}\right)$ does not contain any nontrivial subgroup. Then, $\mathrm{R}(\mathcal{O})$ is a stiff PRO.

Proof. As any operad, $\mathcal{O}$ is the quotient by a certain operadic congruence $\equiv$ of the free operad generated by a certain set of generators $G$. It follows directly from the definition of the construction $\mathrm{R}$ that the PRO $\mathrm{R}(\mathcal{O})$ is the quotient by the congruence of PROs $\equiv^{\prime}$ of the free PRO generated by $G^{\prime}$ where

$$
G^{\prime}(p, q):= \begin{cases}G(p) & \text { if } p \geqslant 1 \text { and } q=1 \\ \emptyset & \text { otherwise }\end{cases}
$$

and $\equiv^{\prime}$ is the finest congruence of PROs satisfying $x \equiv^{\prime} y$ for any relation $x \equiv y$ between elements $x$ and $y$ of the free operad generated by $G$. Since by hypothesis $\left(\mathcal{O}(1), \circ_{1}\right)$ does not contain any nontrivial subgroup, for all elements $x$ and $y$ of $\mathcal{O}(1) \backslash\{\mathbb{1}\}, x \circ_{1} y \neq \mathbb{1}$. Then, $\equiv^{\prime}$ satisfies (C2). Moreover, by definition of $\mathrm{R}, \equiv^{\prime}$ satisfies $(C 3)$. Hence, $\mathrm{R}(\mathcal{O})$ is a stiff PRO.

2.4.2. From monoids to stiff PROs. Any monoid $\mathcal{M}$ can be seen as an operad concentrated in arity one. Then, starting from a monoid $\mathcal{M}$, one can construct a $\mathrm{PRO} B(\mathcal{M})$ by applying the construction $\mathrm{R}$ on $\mathcal{M}$ seen as an operad.

This construction can be rephrased as follows. We have $\mathrm{B}(\mathcal{M})=\sqcup_{p \geqslant 0} \sqcup_{q \geqslant 0} \mathrm{~B}(\mathcal{M})(p, q)$ where

$$
\mathrm{B}(\mathcal{M})(p, q)= \begin{cases}\left\{x_{1} \ldots x_{p}: x_{i} \in M \text { for all } i \in[p]\right\} & \text { if } p=q, \\ \emptyset & \text { otherwise. }\end{cases}
$$


The horizontal composition of $\mathrm{B}(\mathcal{M})$ is the concatenation of sequences and the vertical composition $\circ: \mathrm{B}(\mathcal{M})(p, p) \times \mathrm{B}(\mathcal{M})(p, p) \rightarrow \mathrm{B}(\mathcal{M})(p, p)$ of $\mathrm{B}(\mathcal{M})$ satisfies, for any $x_{1} \ldots x_{p} \in \mathrm{B}(\mathcal{M})(p, p)$ and $y_{1} \ldots y_{q} \in \mathrm{B}(\mathcal{M})(q, q)$,

$$
x_{1} \ldots x_{p} \circ y_{1} \ldots y_{p}=\left(x_{1} \bullet y_{1}\right) \ldots\left(x_{p} \bullet y_{p}\right),
$$

where $\bullet$ is the product of $\mathcal{M}$.

For instance, if $\mathcal{M}$ is the additive monoid of natural numbers, the $\mathrm{PRO} B(\mathcal{M})$ contains all words over $\mathbb{N}$. The horizontal composition of $\mathrm{B}(\mathcal{M})$ is the concatenation of words, and the vertical composition of $\mathrm{B}(\mathcal{M})$, defined only on words with a same length, is the componentwise addition of their letters.

Proposition 2.16. Let $\mathcal{M}$ be a monoid that does not contain any nontrivial subgroup. Then, $\mathrm{B}(\mathcal{M})$ is a stiff PRO.

Proof. Since $\mathcal{M}$ does not contain any nontrivial subgroup, seen as an operad, the elements of arity one of $\mathcal{M}$ do not contain any nontrivial subgroup. Hence, by definition of the construction B passing by $\mathrm{R}$ and by Proposition $2.15, \mathrm{~B}(\mathcal{M})$ is a stiff PRO.

2.4.3. The natural Hopf algebra of an operad. We call abelianization of a bialgebra $\mathcal{H}$ the quotient of $\mathcal{H}$ by the bialgebra ideal spanned by the $x \cdot y-y \cdot x$ for all $x, y \in \mathcal{H}$.

Here is the link between our construction $\mathrm{H}$ and the construction $H$.

Proposition 2.17. Let $\mathcal{O}$ be an operad such that the monoid $\left(\mathcal{O}(1), \circ_{1}\right)$ does not contain any nontrivial subgroup. Then, the bialgebra $H(\mathcal{O})$ is the abelianization of $\mathrm{H}(\mathrm{R}(\mathcal{O}))$.

Proof. By Proposition 2.15, $\mathrm{R}(\mathcal{O})$ is a stiff PRO, and then, by Theorem 2.13, $\mathrm{H}(\mathrm{R}(\mathcal{O})$ ) is a bialgebra. By construction, this bialgebra is freely generated by the $\mathbf{T}_{x}$ where $x \in \mathcal{O}$. Hence, the map $\phi: \mathrm{H}(\mathrm{R}(\mathcal{O})) \rightarrow H(\mathcal{O})$ defined for any $x \in \mathcal{O}$ by $\phi\left(\mathbf{T}_{x}\right):=T_{x}$ can be uniquely extended into a bialgebra morphism, which we denote also by $\phi$. Since $H(\mathcal{O})$ is generated by the $T_{x}$ where $x \in \mathcal{O}, \phi$ is surjective. Directly from the definition of the construction $H$, we observe that the kernel of $\phi$ is the bialgebra ideal $I$ spanned by the $\mathbf{T}_{x * y}-\mathbf{T}_{y * x}$ for all $x, y \in \mathrm{R}(\mathcal{O})$. Then, the associated map $\phi_{I}: \mathrm{H}(\mathrm{R}(\mathcal{O})) /_{I} \rightarrow H(\mathcal{O})$ is a bialgebra isomorphism.

\section{Examples of application of the COnstruction}

We conclude this paper by presenting examples of application of the construction $\mathrm{H}$. The PROs considered in this section fit into the diagram represented by Figure 1 and the obtained Hopf algebras fit into the diagram represented by Figure 2.

3.1. Hopf algebras of forests. We present here the construction of two Hopf algebras of forests, one depending on a nonnegative integer $\gamma$, and with different gradings. The PRO we shall define in this section will intervene in the next examples. 


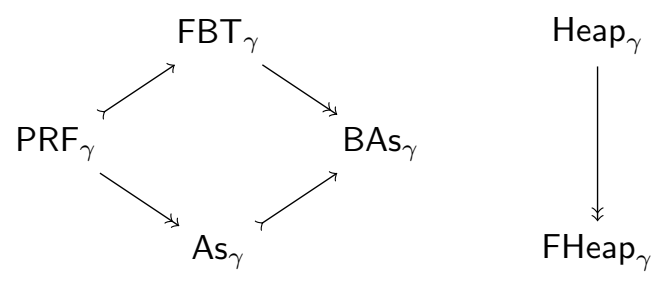

FiguRE 1. Diagram of PROs where arrows $\rightarrow$ (resp. $\rightarrow$ ) are injective (resp. surjective) PRO morphisms. The parameter $\gamma$ is a positive integer. When $\gamma=0$, $\mathrm{PRF}_{0}=\mathrm{As}_{0}=$ Heap $_{0}=\mathrm{FHeap}_{0}$ and $\mathrm{FBT}_{0}=\mathrm{BAs}_{0}$.
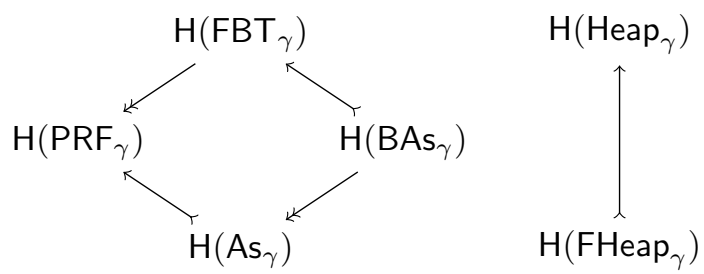

FiguRE 2. Diagram of combinatorial Hopf algebras where arrows $\longmapsto$ (resp. $\rightarrow$ ) are injective (resp. surjective) PRO morphisms. The parameter $\gamma$ is a positive integer. When $\gamma=0, \mathrm{H}\left(\mathrm{PRF}_{0}\right)=\mathrm{H}\left(\mathrm{As}_{0}\right)=\mathrm{H}\left(\mathrm{Heap}_{0}\right)=\mathrm{H}\left(\mathrm{FHeap}_{0}\right)$ and $\mathrm{H}\left(\mathrm{FBT}_{0}\right)=\mathrm{H}\left(\mathrm{BAs}_{0}\right)$.

3.1.1. PRO of forests with a fixed arity. Let $\gamma$ be a nonnegative integer and $\mathrm{PRF}_{\gamma}$ be the free PRO generated by $G:=G(\gamma+1,1):=\{\mathrm{a}\}$, with the grading $\omega$ defined by $\omega(\mathrm{a}):=1$. Any prograph $x$ of $\mathrm{PRF}_{\gamma}$ can be seen as a planar forest of planar rooted trees with only internal nodes of arity $\gamma+1$. Since the reduced elements of $\mathrm{PRF}_{\gamma}$ have no wire, they are encoded by forests of nonempty trees.

3.1.2. Hopf algebra. By Theorem 2.3 and Proposition 2.5, $\mathrm{H}\left(\mathrm{PRF}_{\gamma}\right)$ is a combinatorial Hopf algebra. By Proposition 2.4, as an algebra, $\mathrm{H}\left(\mathrm{PRF}_{\gamma}\right)$ is freely generated by the $\mathbf{S}_{T}$, where the $T$ are nonempty planar rooted trees with only internal nodes of arity $\gamma+1$. Its bases are indexed by planar forests of such trees where the degree of a basis element $\mathbf{S}_{F}$ is the number of internal nodes of $F$.

Notice that the bases of $\mathrm{H}\left(\mathrm{PRF}_{0}\right)$ are indexed by forests of linear trees and that $\mathrm{H}\left(\mathrm{PRF}_{0}\right)$ and Sym are trivially isomorphic as combinatorial Hopf algebras.

3.1.3. Coproduct. By definition of the construction $\mathrm{H}$, the coproduct of $\mathrm{H}\left(\mathrm{PRF}_{\gamma}\right)$ is given on a generator $\mathbf{S}_{T}$ by

$$
\Delta\left(\mathbf{S}_{T}\right)=\sum_{T^{\prime} \in \operatorname{Adm}(T)} \mathbf{S}_{T^{\prime}} \otimes \mathbf{S}_{T / T^{\prime}},
$$

where $\operatorname{Adm}(T)$ is the set of admissible cuts of $T$, that is, the empty tree or the subtrees of $T$ containing the root of $T$ and where $T / T^{\prime}$ denotes the forest consisting in the maximal subtrees 
of $T$ whose roots are leaves of $T^{\prime}$, by respecting the order of these leaves in $T^{\prime}$ and by removing the empty trees. For instance, we have

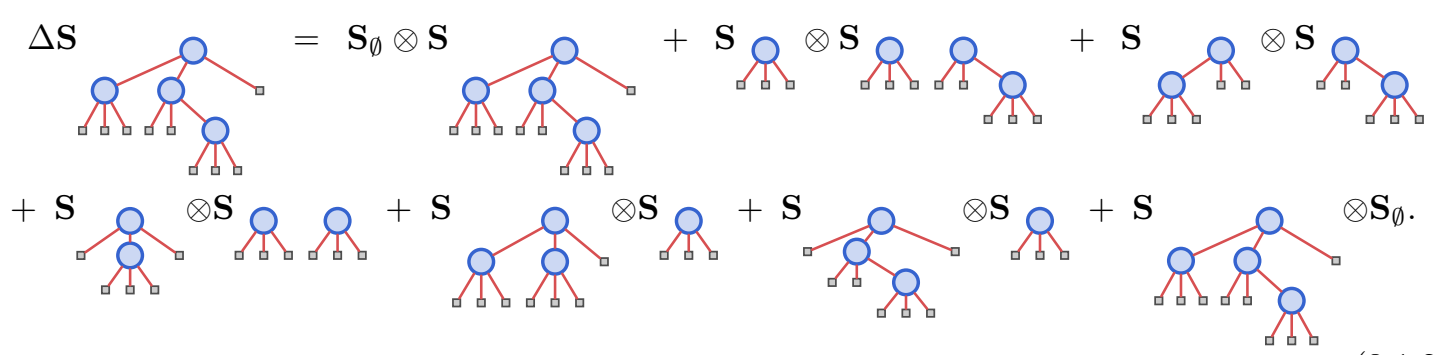

This coproduct is similar to the one of the noncommutative Connes-Kreimer Hopf algebra CK [CK98]. The main difference between $\mathbf{H}\left(\mathrm{PRF}_{\gamma}\right)$ and $\mathbf{C K}$ lies in the fact that in a coproduct of $\mathbf{C K}$, the admissible cuts can change the arity of some internal nodes; it is not the case in $\mathrm{H}\left(\mathrm{PRF}_{\gamma}\right)$ because for any $T^{\prime} \in \operatorname{Adm}(T)$, any internal node $x$ of $T^{\prime}$ has the same arity as it has in $T$.

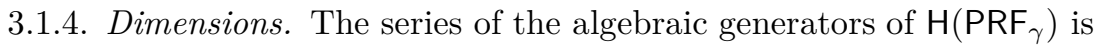

$$
G(t):=\sum_{n \geqslant 1} \frac{1}{n \gamma+1}\left(\begin{array}{c}
n(\gamma+1) \\
n
\end{array}\right) t^{n}
$$

since its coefficients are the Fuss-Catalan numbers, counting planar rooted trees with $n$ internal nodes of arity $\gamma+1$. Since $\mathrm{H}\left(\mathrm{PRF}_{\gamma}\right)$ is free as an algebra, its Hilbert series is $H(t):=\frac{1}{1-G(t)}$.

The first dimensions of $\mathrm{H}\left(\mathrm{PRF}_{1}\right)$ are

$$
1,1,3,10,35,126,462,1716,6435,24310,92378
$$

and those of $\mathrm{H}\left(\mathrm{PRF}_{2}\right)$ are

$$
1,4,19,98,531,2974,17060,99658,590563,3540464,21430267 .
$$

The first sequence is listed in [Slo] as Sequence A001700 and the second as Sequence A047099.

3.1.5. PRO of general forests. We denote by $\mathrm{PRF}_{\infty}$ the free $\mathrm{PRO}$ generated by $G:=\sqcup_{n} \geqslant 1 G(n, 1):=$ $\sqcup_{n \geqslant 1}\left\{\mathrm{a}_{n}\right\}$. Any prograph $x$ of $\mathrm{PRF}_{\infty}$ can be seen as a planar forest of planar rooted trees. Since the reduced elements of $\mathrm{PRF}_{\infty}$ have no wire, they are encoded by forests of nonempty trees. Observe that for any nonnegative integer $\gamma, \mathrm{PRF}_{\gamma}$ is a sub-PRO of $\mathrm{PRF}_{\infty}$. 
3.1.6. Hopf algebra. By Theorem 2.3, $\mathrm{H}\left(\mathrm{PRF}_{\infty}\right)$ is a bialgebra. By Proposition 2.4, as an algebra, $\mathrm{H}\left(\mathrm{PRF}_{\infty}\right)$ is freely generated by the $\mathbf{S}_{T}$, where the $T$ are nonempty planar rooted trees. Its bases are indexed by planar forests of such trees. Besides, by Proposition 2.8, since $\mathrm{PRF}_{\gamma}$ is generated by a subset of the generators of $\mathrm{PRF}_{\infty}, \mathrm{H}\left(\mathrm{PRF}_{\gamma}\right)$ is a bialgebra quotient of $\mathrm{H}\left(\mathrm{PRF}_{\infty}\right)$. Moreover, the coproduct of $\mathrm{H}\left(\mathrm{PRF}_{\infty}\right)$ satisfies (3.1.1).

To turn $\mathrm{H}\left(\mathrm{PRF}_{\infty}\right)$ into a combinatorial Hopf algebra, we cannot consider the grading $\omega$ defined by $\omega\left(\mathrm{a}_{n}\right):=1$ because there would be infinitely many elements of degree 1 . Therefore, we consider on $\mathrm{H}\left(\mathrm{PRF}_{\infty}\right)$ the grading $\omega$ defined by $\omega\left(\mathrm{a}_{n}\right):=n$. In this way, the degree of a basis element $\mathbf{S}_{F}$ is the number of edges of the forest $F$. By Proposition 2.5, $\mathrm{H}\left(\mathrm{PRF}_{\infty}\right)$ is a combinatorial Hopf algebra.

3.1.7. Dimensions. The series of the algebraic generators of $H\left(\mathrm{PRF}_{\infty}\right)$ is

$$
G(t):=\sum_{n \geqslant 1} \frac{1}{n+1}\left(\begin{array}{c}
2 n \\
n
\end{array}\right) t^{n}
$$

since its coefficients are the Catalan numbers, counting planar rooted trees with $n$ edges. As $\mathrm{H}\left(\mathrm{PRF}_{\infty}\right)$ is free as an algebra, its Hilbert series is

$$
H(t):=\frac{1}{1-G(t)}=1+\sum_{n \geqslant 1} \frac{1}{2}\left(\begin{array}{c}
2 n \\
n
\end{array}\right) t^{n}
$$

The dimensions of $\mathrm{H}\left(\mathrm{PRF}_{\infty}\right)$ are then the same as the dimensions of $\mathrm{H}\left(\mathrm{PRF}_{1}\right)$ (see (3.1.4)).

3.2. The Faà di Bruno algebra and its deformations. We shall give here a method to construct the Hopf algebras $\mathbf{F d B}_{\gamma}$ of Foissy [Foi08] from our construction $\mathbf{H}$ in the case where $\gamma$ is a nonnegative integer.

3.2.1. Associative PRO. Let $\gamma$ be a nonnegative integer and $\mathrm{As}_{\gamma}$ be the quotient of $\mathrm{PRF}_{\gamma}$ by the finest congruence $\equiv$ satisfying

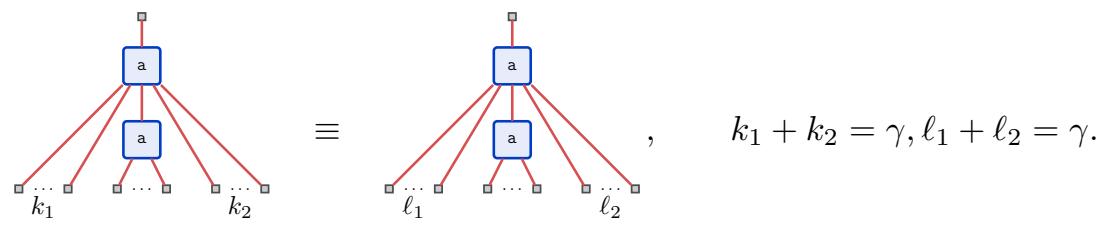

We can observe that $\mathrm{As}_{\gamma}$ is a stiff $\mathrm{PRO}$ because $\equiv$ satisfies $(C 2)$ and $(C 3)$ and that $\mathrm{As}_{0}=\mathrm{PRF}_{0}$. Moreover, observe that, when $\gamma \geqslant 1$, there is in $A s_{\gamma}$ exactly one indecomposable element of arity $n \gamma+1$ for any $n \geqslant 0$. We denote by $\alpha_{n}$ this element. We consider on $\mathrm{As}_{\gamma}$ the grading $\omega$ inherited from the one of $\mathrm{PRT}_{\gamma}$. This grading is still well-defined in $\mathrm{As}_{\gamma}$ since any $\equiv$-equivalence class contains prographs of a same degree and satisfies, for all $n \geqslant 0, \omega\left(\alpha_{n}\right)=n$. Any element of $\mathbf{A s}_{\gamma}$ is then a word $\alpha_{k_{1}} \ldots \alpha_{k_{\ell}}$ and can be encoded by a word of nonnegative integers $k_{1} \ldots k_{\ell}$. Since the reduced elements of $\mathrm{As}_{\gamma}$ have no wire, they are encoded by words of positive integers. 
3.2.2. Hopf algebra. By Theorem 2.13 and Proposition 2.5, $\mathrm{H}\left(\mathrm{As}_{\gamma}\right)$ is a combinatorial Hopf algebra. As an algebra, $\mathbf{H}\left(\mathrm{As}_{\gamma}\right)$ is freely generated by the $\mathbf{T}_{n}, n \geqslant 1$, and its bases are indexed by words of positive integers where the degree of a basis element $\mathbf{T}_{k_{1} \ldots k_{\ell}}$ is $k_{1}+\cdots+k_{\ell}$.

3.2.3. Coproduct. Since any element $\alpha_{n}$ of As $\boldsymbol{s}_{\gamma}$ decomposes into $\alpha_{n}=x \circ y$ if and only if $x=\alpha_{k}$ and $y=\alpha_{i_{1}} \ldots \alpha_{i_{k \gamma+1}}$ with $i_{1}+\cdots+i_{k \gamma+1}=n-k$, by Proposition 2.12, for any $n \geqslant 1$, the coproduct of $\mathrm{H}\left(\mathrm{As}_{\gamma}\right)$ expresses as

$$
\Delta\left(\mathbf{T}_{n}\right)=\sum_{k=0}^{n} \mathbf{T}_{k} \otimes\left(\sum_{i_{1}+\cdots+i_{k \gamma+1}=n-k} \mathbf{T}_{i_{1}} \ldots \mathbf{T}_{i_{k \gamma+1}}\right),
$$

where $\mathbf{T}_{0}$ is identified with the unity $\mathbf{T}_{\epsilon}$ of $\mathbf{H}\left(\mathrm{As}_{\gamma}\right)$. For instance, in $\mathbf{H}\left(\mathrm{As}_{1}\right)$, we have

$$
\begin{aligned}
& \Delta\left(\mathbf{T}_{3}\right)=\mathbf{T}_{0} \otimes \mathbf{T}_{3}+\mathbf{T}_{1} \otimes\left(\mathbf{T}_{0} \mathbf{T}_{2}+\mathbf{T}_{1} \mathbf{T}_{1}+\mathbf{T}_{2} \mathbf{T}_{0}\right) \\
& +\mathbf{T}_{2} \otimes\left(\mathbf{T}_{0} \mathbf{T}_{0} \mathbf{T}_{1}+\mathbf{T}_{0} \mathbf{T}_{1} \mathbf{T}_{0}+\mathbf{T}_{1} \mathbf{T}_{0} \mathbf{T}_{0}\right)+\mathbf{T}_{3} \otimes\left(\mathbf{T}_{0} \mathbf{T}_{0} \mathbf{T}_{0} \mathbf{T}_{0}\right) \\
& =\mathbf{T}_{\epsilon} \otimes \mathbf{T}_{3}+2 \mathbf{T}_{1} \otimes \mathbf{T}_{2}+\mathbf{T}_{1} \otimes \mathbf{T}_{11}+3 \mathbf{T}_{2} \otimes \mathbf{T}_{1}+\mathbf{T}_{3} \otimes \mathbf{T}_{\epsilon},
\end{aligned}
$$

and in $\mathrm{H}\left(\mathrm{As}_{2}\right)$, we have

$$
\begin{aligned}
& \Delta\left(\mathbf{T}_{3}\right)=\mathbf{T}_{0} \otimes \mathbf{T}_{3}+\mathbf{T}_{1} \otimes\left(\mathbf{T}_{0} \mathbf{T}_{0} \mathbf{T}_{2}+\mathbf{T}_{0} \mathbf{T}_{2} \mathbf{T}_{0}+\mathbf{T}_{2} \mathbf{T}_{0} \mathbf{T}_{0}+\mathbf{T}_{0} \mathbf{T}_{1} \mathbf{T}_{1}+\mathbf{T}_{1} \mathbf{T}_{0} \mathbf{T}_{1}+\mathbf{T}_{1} \mathbf{T}_{1} \mathbf{T}_{0}\right) \\
& +\mathbf{T}_{2} \otimes\left(\mathbf{T}_{0} \mathbf{T}_{0} \mathbf{T}_{0} \mathbf{T}_{0} \mathbf{T}_{1}+\mathbf{T}_{0} \mathbf{T}_{0} \mathbf{T}_{0} \mathbf{T}_{1} \mathbf{T}_{0}+\mathbf{T}_{0} \mathbf{T}_{0} \mathbf{T}_{1} \mathbf{T}_{0} \mathbf{T}_{0}+\mathbf{T}_{0} \mathbf{T}_{1} \mathbf{T}_{0} \mathbf{T}_{0} \mathbf{T}_{0}+\mathbf{T}_{1} \mathbf{T}_{0} \mathbf{T}_{0} \mathbf{T}_{0} \mathbf{T}_{0}\right) \\
& +\mathbf{T}_{3} \otimes \mathbf{T}_{0} \mathbf{T}_{0} \mathbf{T}_{0} \mathbf{T}_{0} \mathbf{T}_{0} \mathbf{T}_{0} \mathbf{T}_{0} \\
& =\mathbf{T}_{\epsilon} \otimes \mathbf{T}_{3}+3 \mathbf{T}_{1} \otimes \mathbf{T}_{2}+3 \mathbf{T}_{1} \otimes \mathbf{T}_{11}+5 \mathbf{T}_{2} \otimes \mathbf{T}_{1}+\mathbf{T}_{3} \otimes \mathbf{T}_{\epsilon}
\end{aligned}
$$

\subsubsection{Isomorphism with the deformation of the noncommutative Faà di Bruno Hopf algebra.}

Theorem 3.1. For any nonnegative integer $\gamma$, the Hopf algebra $\mathrm{H}\left(\mathrm{As}_{\gamma}\right)$ is the deformation of the noncommutative Faà di Bruno Hopf algebra $\mathbf{F d B}_{\gamma}$.

Proof. Let us set $\sigma_{1}:=\sum_{n \geqslant 0} \mathbf{T}_{n}$. By (3.2.2), we have

$$
\begin{aligned}
\Delta\left(\sigma_{1}\right) & =\sum_{n \geqslant 0} \sum_{k=0}^{n} \mathbf{T}_{k} \otimes\left(\sum_{i_{1}+\cdots+i_{k \gamma+1}=n-k} \mathbf{T}_{i_{1}} \ldots \mathbf{T}_{i_{k \gamma+1}}\right) \\
& =\sum_{k \geqslant 0} \mathbf{T}_{k} \otimes\left(\sum_{n \geqslant k} \sum_{i_{1}+\cdots+i_{k \gamma+1}=n-k} \mathbf{T}_{i_{1}} \ldots \mathbf{T}_{i_{k \gamma+1}}\right) \\
& =\sum_{k \geqslant 0} \mathbf{T}_{k} \otimes\left(\sum_{i_{1}+\cdots+i_{k \gamma+1} \geqslant 0} \mathbf{T}_{i_{1}} \ldots \mathbf{T}_{i_{k \gamma+1}}\right) \\
& =\sum_{k \geqslant 0} \mathbf{T}_{k} \otimes\left(\sum_{i \geqslant 0} \mathbf{T}_{i}\right)^{k \gamma+1} \\
& =\sum_{k \geqslant 0} \mathbf{T}_{k} \otimes \sigma_{1}^{k \gamma+1},
\end{aligned}
$$


showing that for any $n \geqslant 0, \Delta\left(\mathbf{T}_{n}\right)$ is the homogeneous component of degree $n$ in $\sum_{k \geqslant 0} \mathbf{T}_{k} \otimes$ $\sigma_{1}^{k \gamma+1}$.

Therefore, by the definition of the coproduct of $\mathbf{F d B}$ (see (1.1.5)), the map $\mathbf{T}_{n} \mapsto \mathbf{S}_{n}$ from $\mathbf{H}\left(\mathrm{As}_{\gamma}\right)$ to $\mathbf{F d B}_{\gamma}$ is an isomorphism of Hopf algebras.

3.3. Hopf algebra of forests of bitrees. Let us describe here a general construction on PROs. If $G$ is a bigraded set of the form $G=\sqcup_{p \geqslant 1} \sqcup_{q \geqslant 1} G(p, q)$, we denote by $G^{\text {op }}$ the bigraded set defined by

$$
G^{\mathrm{op}}(p, q):=G(q, p), \quad p, q \geqslant 1 .
$$

From a geometrical point of view, any elementary prograph over $G^{\text {op }}$ is obtained by reversing from bottom to top an elementary prograph over $G$. We moreover denote by rev : $\operatorname{Free}\left(G^{\mathrm{op}}\right) \rightarrow \operatorname{Free}(G)$ the bijection sending any prograph $x$ of $\operatorname{Free}\left(G^{\text {op }}\right)$ to the prograph $\operatorname{rev}(x)$ of $\operatorname{Free}(G)$ obtained by reversing $x$ from bottom to top.

Now, given a PRO $\mathcal{P}:=\operatorname{Free}(G) / \equiv$, we define $\mathrm{S}(\mathcal{P})$ as the PRO

$$
\mathrm{S}(\mathcal{P}):=\text { Free }\left(G \sqcup G^{\mathrm{op}}\right) / \cong,
$$

where $\cong$ is the finest congruence of $\operatorname{Free}\left(G \sqcup G^{\mathrm{op}}\right)$ satisfying

$$
x \cong y \quad \text { if }(x, y \in \operatorname{Free}(G) \text { and } x \equiv y) \quad \text { or } \quad\left(x, y \in \operatorname{Free}\left(G^{\mathrm{op}}\right) \text { and } \operatorname{rev}(x) \equiv \operatorname{rev}(y)\right) .
$$

Notice that in this definition, we consider $\operatorname{Free}(G)$ and $\operatorname{Free}\left(G^{\mathrm{op}}\right)$ as sub-PROs of $\operatorname{Free}\left(G \sqcup G^{\mathrm{op}}\right)$ in an obvious way. Notice also that if $\mathcal{P}$ is a free PRO Free $(G)$, then the congruence $\equiv$ is trivial, so that $\cong$ is also trivial, and $\mathrm{S}(\mathcal{P})=\operatorname{Free}\left(G \sqcup G^{\text {op }}\right)$. Besides, as an other immediate property of this construction, remark that when $\mathcal{P}$ is a stiff $\mathrm{PRO}$, the congruence $\cong$ satisfies $\left(C_{2}\right)$ and $\left(C_{3}\right)$, and then, $\mathrm{S}(\mathcal{P})$ is a stiff PRO.

We shall present here two Hopf algebras coming from the construction $\mathrm{S}$ applied on $\mathrm{PRF}_{\gamma}$ and $\mathrm{As}_{\gamma}$.

3.3.1. PRO of forests of bitrees. Let $\gamma$ be a nonnegative integer and $\mathrm{FBT}_{\gamma}$ be the free PRO generated by $G:=G(\gamma+1,1) \sqcup G(1, \gamma+1)$ where $G(\gamma+1,1):=\{\mathrm{a}\}$ and $G(1, \gamma+1):=\{\mathrm{b}\}$, with the grading $\omega$ defined by $\omega(\mathrm{a}):=\omega(\mathrm{b}):=1$. One has $\mathrm{S}\left(\mathrm{PRF}_{\gamma}\right)=\mathrm{FBT}_{\gamma}$. Any prograph $x$ of $\mathrm{FBT}_{\gamma}$ can be seen as a forest of $\gamma$-bitrees, that are labeled planar trees where internal nodes labeled by a have $\gamma+1$ children and one parent, and the internal nodes labeled by $\mathrm{b}$ have one child and $\gamma+1$ parents. Since the reduced elements of $\mathrm{FBT}_{\gamma}$ have no wire, they are encoded by forests of nonempty $\gamma$-bitrees.

3.3.2. Hopf algebra. By Theorem 2.3 and Proposition 2.5, $\mathrm{H}\left(\mathrm{FBT}_{\gamma}\right)$ is a combinatorial Hopf algebra. By Proposition 2.4, as an algebra, $\left.\mathrm{H}_{(\mathrm{FBT}}\right)$ is freely generated by the $\mathbf{S}_{T}$, where the $T$ are nonempty $\gamma$-bitrees. Its bases are indexed by planar forests of such bitrees where the degree of a basis element $\mathbf{S}_{F}$ is the total number of internal nodes in the bitrees of $F$. Moreover, by Proposition 2.8, since $\mathrm{PRF}_{\gamma}$ is generated by a subset of the generators of $\mathrm{FBT}_{\gamma}, \mathrm{H}\left(\mathrm{PRF}_{\gamma}\right)$ is a quotient bialgebra of $\mathrm{H}\left(\mathrm{FBT}_{\gamma}\right)$. 
3.3.3. Coproduct. The coproduct of $\mathrm{H}\left(\mathrm{FBT}_{\gamma}\right)$ can be described, like the one of $\mathbf{C K}$ on forests, by means of admissible cuts on forests of $\gamma$-bitrees. We have for instance

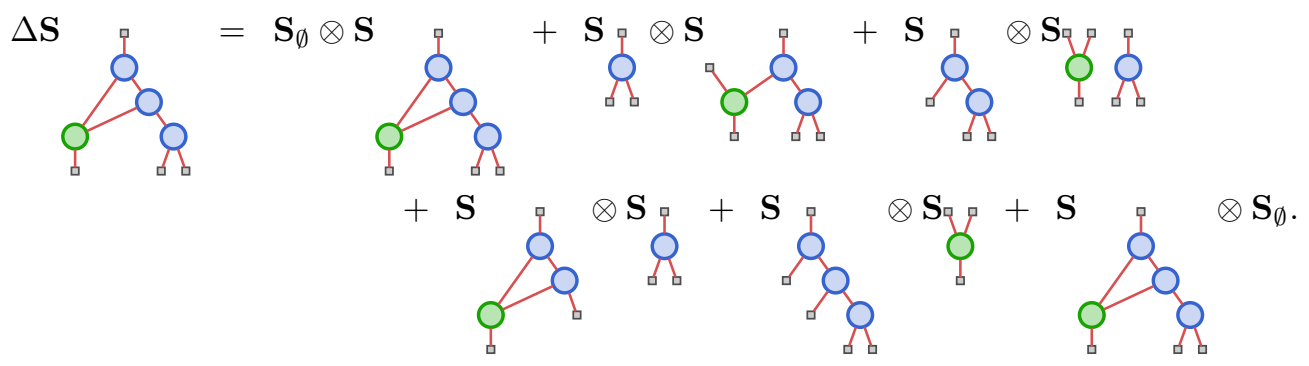

3.3.4. Dimensions. We only know the dimensions of $\mathbf{H}\left(\mathrm{FBT}_{\gamma}\right)$ when $\gamma=0$. In this case, 0 -bitrees of size $n$ are linear trees and can hence be seen as words of length $n$ on the alphabet $\{\mathrm{a}, \mathrm{b}\}$. Therefore, as $\mathrm{H}\left(\mathrm{FBT}_{\gamma}\right)$ is free as an algebra, the bases of $\mathrm{H}\left(\mathrm{FBT}_{0}\right)$ are indexed by multiwords on $\{a, b\}$ and its Hilbert series is

$$
H(t):=1+\sum_{n \geqslant 1} 2^{2 n-1} t^{n}
$$

3.3.5. PRO of biassociative operators and its Hopf algebra. Let $\mathrm{BAs}_{\gamma}$ be the quotient of $\mathrm{FBT}_{\gamma}$ by the finest congruence $\equiv$ satisfying

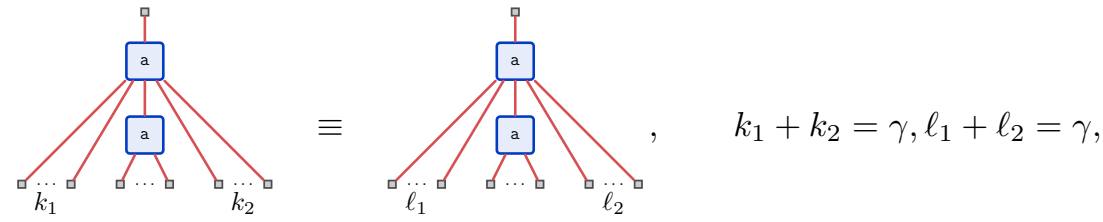

and

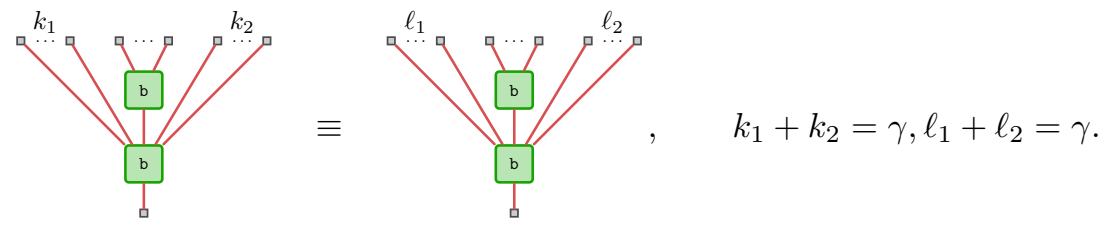

We can observe that $\mathrm{BAs}_{\gamma}$ is a stiff $\mathrm{PRO}$ because $\equiv$ satisfies $\left(C_{2}\right)$ and $\left(C_{3}\right)$. Notice that $\mathrm{S}\left(\mathrm{As}_{\gamma}\right)=\mathrm{BAs}_{\gamma}$ and $\mathrm{BAs}_{0}=\mathrm{FBT}_{0}$. We consider on $\mathrm{BAs}_{\gamma}$ the grading $\omega$ inherited from the one of $\mathrm{FBT}_{\gamma}$. This grading is still well-defined in $\mathrm{BAs}_{\gamma}$ since any $\equiv$-equivalence class contains prographs of a same degree. Notice that $B A s_{1}$ is very similar to the PRO governing bialgebras (see [Mar08]). Indeed, it only lacks in $\mathrm{BAs}_{1}$ the usual compatibility relation between its two generators. Notice also that the PRO governing bialgebras is not a stiff PRO.

By Theorem 2.13 and Proposition 2.5, $\mathrm{H}\left(\mathrm{BAs}_{\gamma}\right)$ is then a combinatorial Hopf algebra. Moreover, we can observe that $\mathrm{H}\left(\mathrm{As}_{\gamma}\right)$ is a quotient bialgebra of $\mathrm{H}\left(\mathrm{BAs} \mathbf{s}_{\gamma}\right)$. 
3.4. Hopf algebra of heaps of pieces. We present here the construction of a Hopf algebra depending on a nonnegative integer $\gamma$, whose bases are indexed by heaps of pieces.

3.4.1. PRO of heaps of pieces. Let $\gamma$ be a nonnegative integer and $\mathrm{Heap}_{\gamma}$ be the free PRO generated by $G:=G(\gamma+1, \gamma+1):=\{\mathrm{a}\}$, with the grading $\omega$ defined by $\omega(\mathrm{a}):=1$. Any prograph $x$ of Heap ${ }_{\gamma}$ can be seen as a heap of pieces of width $\gamma+1$ (see [Vie86] for some theory about these objects). For instance, the prograph

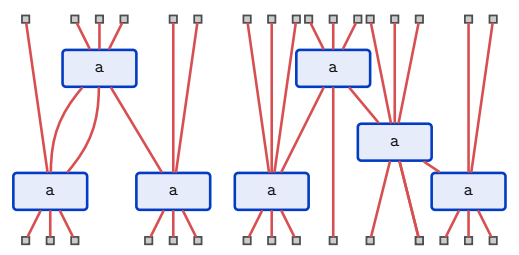

of Heap ${ }_{2}$ is encoded by the heap of pieces of width 3 depicted by

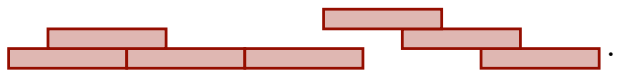

Notice that $\mathrm{Heap}_{0}=\mathrm{PRF}_{0}$. Besides, since the reduced elements of Heap ${ }_{\gamma}$ have no wire, they are encoded by horizontally connected heaps of pieces of width $\gamma+1$.

3.4.2. Hopf algebra. By Theorem 2.3 and Proposition 2.5, $\mathrm{H}\left(\mathrm{Heap}_{\gamma}\right)$ is a combinatorial Hopf algebra. By Proposition 2.4, as an algebra, $\mathbf{H}\left(\mathrm{Heap}_{\gamma}\right)$ is freely generated by the $\mathbf{S}_{\lambda}$ where the $\lambda$ are heaps of pieces that cannot be obtained by juxtaposing two heaps of pieces. Its bases are indexed by horizontally connected heaps of pieces of width $\gamma+1$ where the degree of a basis element $\mathbf{S}_{\Lambda}$ is the number of pieces of $\Lambda$.

3.4.3. Coproduct. The coproduct of $\mathbf{H}\left(\mathrm{Heap}_{\gamma}\right)$ can be described, like the one of $\mathbf{C K}$ on forests, by means of admissible cuts on heaps of pieces. Indeed, if $\Lambda$ is a horizontally connected heap of pieces, by definition of the construction $\mathrm{H}$,

$$
\Delta\left(\mathbf{S}_{\Lambda}\right)=\sum_{\Lambda^{\prime} \in \operatorname{Adm}(\Lambda)} \mathbf{S}_{\Lambda^{\prime}} \otimes \mathbf{S}_{\Lambda / \Lambda^{\prime}}
$$

where $\operatorname{Adm}(\Lambda)$ is the set of admissible cuts of $\Lambda$, that is, the set of heaps of pieces obtained by keeping an upper part of $\Lambda$ and by readjusting it so that it becomes horizontally connected and where $\Lambda / \Lambda^{\prime}$ denotes the heap of pieces obtained by removing from $\Lambda$ the pieces of $\Lambda^{\prime}$ and by readjusting the remaining pieces so that they form an horizontally connected heap of pieces. For instance, in $\mathrm{H}\left(\mathrm{Heap}_{1}\right)$, we have

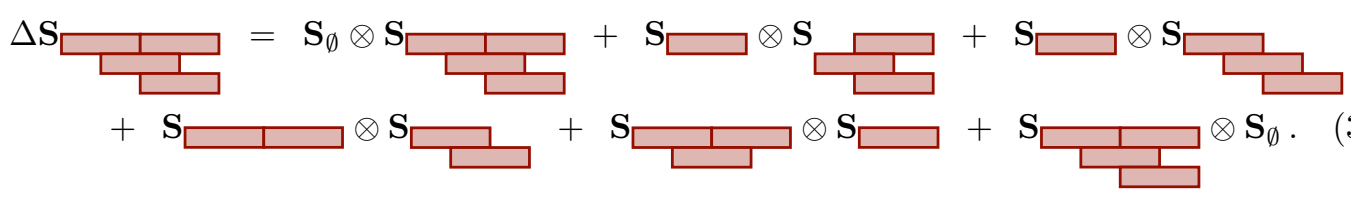




\subsubsection{Dimensions.}

Proposition 3.2. For any nonnegative integer $\gamma$, the Hilbert series $C_{\gamma}(t)$ of $\mathrm{H}\left(\mathrm{Heap}_{\gamma}\right)$ satisfies $C_{\gamma}(t)=\sum_{n \geqslant 0} C_{\gamma, n}(t)$, where

$$
\begin{gathered}
C_{\gamma, n}(t):=P_{\gamma, n}(t)-\sum_{k=0}^{n-1} C_{\gamma, k}(t) P_{\gamma, n-k-1}(t), \\
P_{\gamma, n}(t):=\frac{1}{F_{\gamma, n}(t)}
\end{gathered}
$$

and

$$
F_{\gamma, n}(t):= \begin{cases}1 & \text { if } n \leqslant \gamma \\ F_{\gamma, n-1}(t)-t F_{\gamma, n-\gamma-1}(t) & \text { otherwise }\end{cases}
$$

Proof. The proof of this statement uses the Inversion Lemma of Viennot [Vie86] and some ideas employed in [BMR02] for the enumeration of the so-called connected heaps.

The first ingredient consists in the alternating generating series

$$
\sum_{x=\mathbb{1}_{p_{1}} * \mathrm{a} * \mathbb{1}_{p_{2}} * \cdots * \mathbb{1}_{p_{\ell}} * \mathrm{a} * \mathbb{1}_{p_{\ell+1}} \in \mathrm{Heap}_{\gamma}}(-1)^{\operatorname{deg}(x)} t^{\operatorname{deg}(x)}
$$

of the heaps of pieces of Heap ${ }_{\gamma}$ of height no greater than 1 (called trivial heaps in [Vie86] and [BMR02]). This series is obviously $F_{\gamma, n}(t)$. By the Inversion Lemma, we have that $P_{\gamma, n}(t)$ is the generating series of the elements of $\mathrm{Heap}_{\gamma}$ with exactly $n$ inputs (and thus, also $n$ outputs).

Now, to count only the reduced elements of Heap $\gamma$, observe that any element $x$ of Heap with $n$ inputs is either reduced or is of the form $x=y * \mathbb{1}_{1} * z$ where $y$ is a reduced element of Heap $\gamma$ with $k$ inputs and $z$ is an element of $\operatorname{Heap}_{\gamma}$ with $n-k-1$ inputs. Then, we have

$$
P_{\gamma, n}(t)=C_{\gamma, n}(t)+\sum_{k=0}^{n-1} C_{\gamma, k}(t) P_{\gamma, n-k-1}(t),
$$

so that $C_{\gamma, n}(t)$ counts the reduced elements of Heap ${ }_{\gamma}$ with $n$ inputs. Whence the result.

By using Proposition 3.2, one can compute the first dimensions of $\mathrm{H}\left(\mathrm{Heap}_{\gamma}\right)$. The first dimensions of $\mathrm{H}\left(\mathrm{Heap}_{1}\right)$ are

$$
1,1,4,18,85,411,2014,9950,49417,246302,1230623,
$$

and those of $\mathrm{H}\left(\mathrm{Heap}_{2}\right)$ are

$$
1,1,6,42,313,2407,18848,149271,1191092,9553551,76910632 .
$$

Since by Proposition 2.4, $\mathrm{H}\left(\mathrm{Heap}_{\gamma}\right)$ is free as an algebra, the series $G_{\gamma}(t)$ of its algebraic generators satisfies $G_{\gamma}(t)=1-\frac{1}{C_{\gamma}(t)}$. The first dimensions of the algebraic generators of $\mathrm{H}\left(\mathrm{Heap}_{1}\right)$ are

$$
1,3,11,44,184,790,3450,15242,67895,304267,1369761
$$

and those of $\mathrm{H}\left(\mathrm{Heap}_{2}\right)$ are

$$
1,5,31,210,1488,10826,80111,599671,4525573,34357725,262011295 .
$$

Among these four integer sequences, only (3.4.12) is listed in [Slo] as Sequence A059715. 
3.5. Hopf algebra of heaps of friable pieces. From a PRO being a special quotient of Heap ${ }_{\gamma}$, we construct a Hopf algebra structure on the $(\gamma+1)$-st tensor power of the vector space Sym.

3.5.1. PRO of heaps of friable pieces. Let $\gamma$ be a nonnegative integer and $\mathrm{FHeap}_{\gamma}$ be the quotient of Heap $_{\gamma}$ by the finest congruence $\equiv$ satisfying

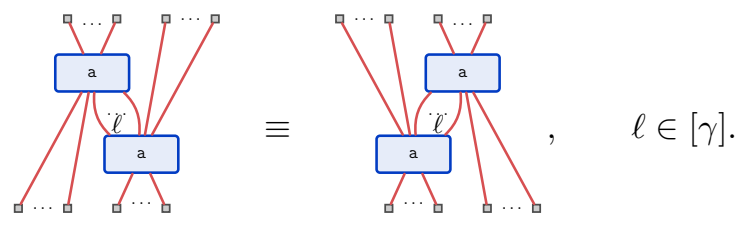

For instance, for $\gamma=2$, the $\equiv$-equivalence class of

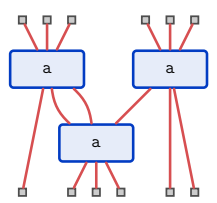

contains exactly the prographs
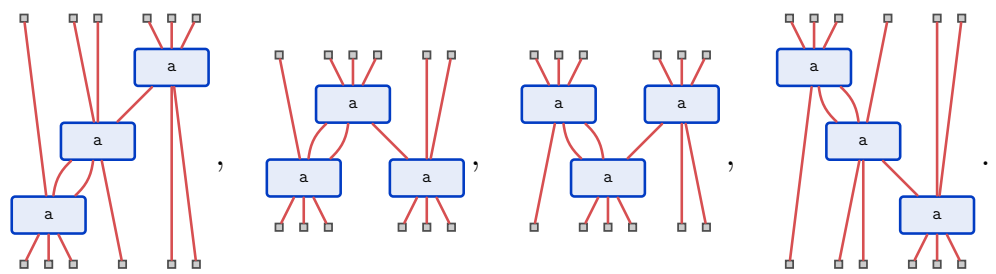

We can observe that FHeap ${ }_{\gamma}$ is a stiff PRO because $\equiv$ satisfies (C2) and (C3) and FHeap $_{0}=$ Heap $_{0}$. We call FHeap F $_{\gamma}$ the PRO of heaps of friable pieces of width $\gamma+1$. This terminology is justified by the following observation. Any piece of width $\gamma+1$ (depicted by $\square$ ) consists in $\gamma+1$ small pieces, called bursts, glued together. This forms a friable piece (depicted, for $\gamma=2$ for instance, by $\square \perp$ ). The congruence $\equiv$ of Heap ${ }_{\gamma}$ can be interpreted by letting all pieces break under gravity, separating the bursts constituting these. For instance, the prographs of (3.5.3), respectively, encoded by the heaps of pieces

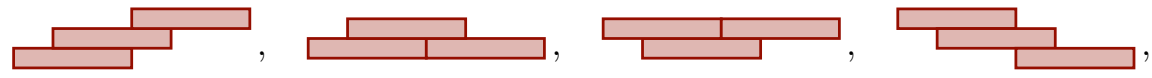

all become the heap of friable pieces

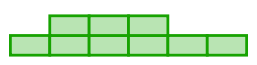

obtained by replacing each piece of any heap of pieces of (3.5.4) by friable pieces.

The grading $\omega$ of $\mathrm{FHeap}_{\gamma}$ is the one inherited from the one of Heap $\mathrm{p}_{\gamma}$. This grading is still well-defined in $\mathrm{Heap}_{\gamma}$ since any $\equiv$-equivalence class contains prographs of a same degree. Since the reduced elements of $\mathrm{FHeap}_{\gamma}$ have no wire, they are encoded by horizontally connected heaps of friable pieces. 


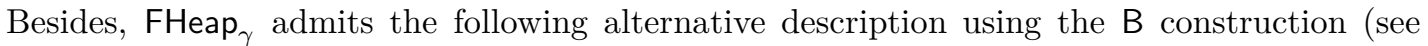

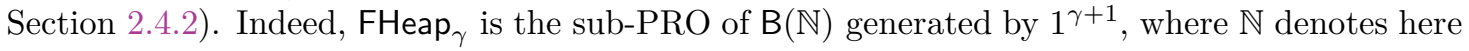
the additive monoid of nonnegative integers and $1^{\gamma+1}$ denotes the sequence of $\gamma+1$ occurrences of $1 \in \mathbb{N}$. The correspondence between heaps of friable pieces and words of integers of this second description is clear since any element $x$ of the sub-PRO of $\mathrm{B}(\mathbb{N})$ generated by $1^{\gamma+1}$ encodes a heap of friable pieces consisting, from left to right, in columns of $x_{i}$ bursts for $i \in[n]$, where $n$ is the length of $x$. For instance, the word 122211 encodes the heap of friable pieces of (3.5.5).

3.5.2. Hopf algebra. By Theorem 2.13 and Proposition 2.5, $\mathrm{H}\left(\mathrm{FHeap}_{\gamma}\right)$ is a combinatorial Hopf subalgebra of $\mathrm{H}\left(\mathrm{Heap}_{\gamma}\right)$. The bases of $\mathrm{H}\left(\mathrm{FHeap}_{\gamma}\right)$ are indexed by horizontally connected heaps of friable pieces of width $\gamma+1$ where the degree of a basis element $\mathbf{T}_{\Lambda}$ is the number of pieces of $\Lambda$.

3.5.3. Coproduct. The coproduct of $\mathrm{H}\left(\mathrm{FHeap}_{\gamma}\right)$ can be described with the aid of the interpretation of $\mathrm{FHeap}_{\gamma}$ as a sub-PRO of $\mathrm{B}(\mathbb{N})$. Indeed, if $\Lambda$ is an horizontally connected heap of friable pieces, by Proposition 2.12,

$$
\Delta\left(\mathbf{T}_{\Lambda}\right)=\sum_{\substack{\Lambda_{1}, \Lambda_{2} \in \mathrm{FHeap}_{\gamma} \\ \Lambda=\Lambda_{1}+\Lambda_{2}}} \mathbf{T}_{\Lambda_{1}^{\prime}} \otimes \mathbf{T}_{\Lambda_{2}^{\prime}},
$$

where $\Lambda_{1}+\Lambda_{2}$ is the heap of friable pieces obtained by stacking $\Lambda_{2}$ onto $\Lambda_{1}$ and where $\Lambda_{1}^{\prime}$ (resp. $\Lambda_{2}^{\prime}$ ) is the readjustment of $\Lambda_{1}$ (resp. $\Lambda_{2}$ ) so that it is horizontally connected. For instance, we have in $\mathrm{H}\left(\mathrm{FHeap}_{1}\right)$

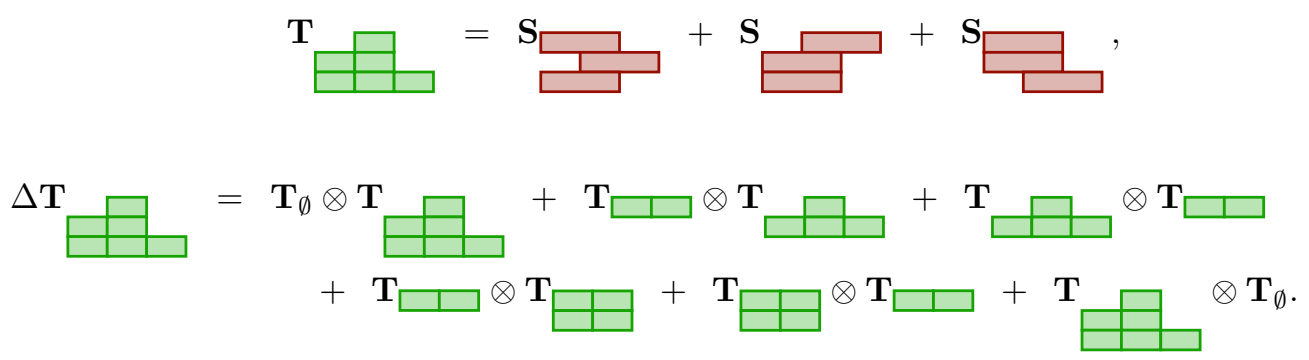

\subsubsection{Dimensions.}

Proposition 3.3. For any nonnegative integer $\gamma$, the $n$-th homogeneous component of $\mathrm{H}\left(\mathrm{FHeap}_{\gamma}\right)$ has dimension $(\gamma+2)^{n-1}$.

Proof. Let us show that there are $(\gamma+2)^{n-1}$ reduced elements in FHeap .

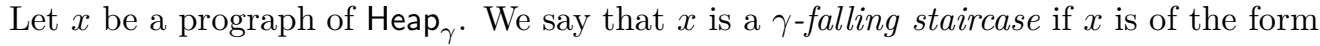

$$
x=\left(\mathbb{1}_{p_{1}} * \mathrm{a} * \mathbb{1}_{q_{1}}\right) \circ\left(\mathbb{1}_{p_{1}+p_{2}} * \mathrm{a} * \mathbb{1}_{q_{2}}\right) \circ \cdots \circ\left(\mathbb{1}_{p_{1}+p_{2}+\cdots+p_{\ell}} * \mathrm{a} * \mathbb{1}_{q_{\ell}}\right),
$$


where $p_{1}=0, q_{\ell}=0$, and $0 \leqslant p_{i} \leqslant \gamma$ for all $2 \leqslant i \leqslant \ell$. Any $\gamma$-falling staircase can be encoded by the sequence $\left(p_{2}, \ldots, p_{\ell}\right)$ involved in its decomposition (3.5.9). For instance, for

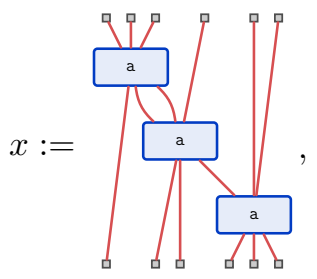

we have $x=\left(\mathbb{1}_{0} * \mathrm{a} * \mathbb{1}_{3}\right) \circ\left(\mathbb{1}_{0+1} * \mathrm{a} * \mathbb{1}_{2}\right) \circ\left(\mathbb{1}_{0+1+2} * \mathrm{a} * \mathbb{1}_{0}\right)$ so that $x$ is a 2 -falling staircase encoded by the sequence $(1,2)$.

Moreover, we say that $x$ is a $\gamma$-standard form if $x$ is an horizontal composition of $\gamma$-falling staircases. Any $\gamma$-standard form can be encoded by the sequence of the sequences encoding, from left to right, its falling staircases.

By definition of FHeap $\gamma$ as a quotient of $\mathrm{Heap}_{\gamma}$, one can observe that two different $\gamma$-standard forms are sent to two different heaps of friable pieces by the canonical surjection Heap $\mathrm{p}_{\gamma} \rightarrow$ FHeap $_{\gamma}$. Besides, one can straightforwardly prove by induction on the degree that any reduced element of Heap $_{\gamma}$ is $\equiv$-equivalent to a $\gamma$-falling staircase.

This shows that the reduced elements of $\mathrm{FHeap}_{\gamma}$ are in bijection with the standard forms of

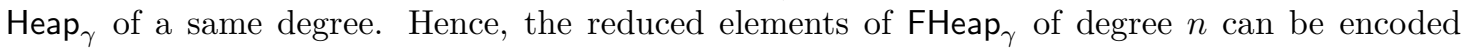
by sequences of $k$ words on the alphabet $\{0\} \cup[\gamma]$, having a total of $n-k$ letters. Whence the result.

3.5.5. Miscellaneous properties. By the dimensions of $\mathrm{H}\left(\mathrm{FHeap}_{\gamma}\right)$ provided by Proposition 3.3, as a graded vector space, $\mathrm{H}\left(\mathrm{FHeap}_{\gamma}\right)$ is the $\gamma+1$-st tensor power of the underlying vector space of Sym. Indeed, the $n$-th homogeneous components of these two spaces have the same dimension. Besides, notice that since $\mathrm{FHeap}_{\gamma}$ is by definition a sub-PRO of the PRO obtained by applying the construction $\mathrm{B}$ on a commutative monoid, $\mathrm{H}\left(\mathrm{FHeap}_{\gamma}\right)$ is cocommutative.

\section{CONCLUDING REMARKS AND PERSPECTIVES}

We have defined a construction $\mathrm{H}$ establishing a new link between the theory of PROs and the theory of combinatorial Hopf algebras, by generalizing a former construction from operads to bialgebras. By the way, we have exhibited the so-called stiff PROs which is the most general class of PROs for which our construction works.

By using $\mathrm{H}$, we have defined some new and recovered some already known combinatorial Hopf algebras by starting with very simple PROs. Nevertheless, we are very far from having exhausted the possibilities, and it would not be surprising that $\mathrm{H}$ could reconstruct some other known Hopf algebras, maybe in an unexpected basis.

Computing the Hilbert series of a combinatorial Hopf algebra is, usually, a routine work. Nevertheless, in the general case, it is very difficult to compute the Hilbert series of $\mathbf{H} \mathcal{P}$ when $\mathcal{P}$ 
is a free PRO. Indeed, this computation requires to know, given a free $\mathrm{PRO} \mathcal{P}$, the series

$$
F_{\mathcal{P}}(t):=\sum_{x \in \operatorname{red}(\mathcal{P})} t^{\operatorname{deg}(x)}
$$

which seems difficult to explicitly describe in general and is not known to the knowledge of the authors.

As an other perspective, it is conceivable to go further in the study of the algebraic structure of the bialgebras obtained by $\mathrm{H}$. The question of the potential autoduality of $\mathrm{H} \mathcal{P}$ depending on some conditions on the PRO $\mathcal{P}$ is noteworthy. A way to solve this problem is to provide enough conditions on $\mathcal{P}$ to endow $\mathrm{H} \mathcal{P}$ with a bidendriform bialgebra structure [Foi07]. In such algebraic structures, there are two products $\prec$ and $\succ$ and two coproducts $\Delta_{\prec}$ and $\Delta_{\succ}$ satisfying some precise axioms. This way to solve this perspective is based upon the fact that any bidendriform bialgebra is free and self-dual as a bialgebra [Foi07].

\section{REFERENCES}

[BFK06] C. Brouder, A. Frabetti, and C. Krattenthaler. Non-commutative Hopf algebra of formal diffeomorphisms. Adv. Math., 200(2):479-524, 2006.

[BMR02] M. Bousquet-Mélou and A. Rechnitzer. Lattice animals and heaps of dimers. Discrete Math., 258(13):235-274, 2002.

[BV73] J. M. Boardman and R. M. Vogt. Homotopy invariant algebraic structures on topological spaces. Lect. Notes Math., 347, 1973.

[Cha08] F. Chapoton. Operads and algebraic combinatorics of trees. Sém. Lothar. Combin., 58, 2008.

[CK98] A. Connes and D. Kreimer. Hopf algebras, renormalization and noncommutative geometry. Comm. Math. Phys., 199:203-242, 1998.

[CL07] F. Chapoton and M. Livernet. Relating two Hopf algebras built from an operad. Int. Math. Res. Notices, (24):Art. ID rnm131, 27, 2007.

[DHT02] G. Duchamp, F. Hivert, and J.-Y. Thibon. Noncommutative Symmetric Functions VI: Free QuasiSymmetric Functions and Related Algebras. Int. J. Algebr. Comput., 12(5):671-717, 2002.

[Foi07] L. Foissy. Bidendriform bialgebras, trees, and free quasi-symmetric functions. J. Pure Appl. Algebra, 209(2):439-459, 2007.

[Foi08] L. Foissy. Faà di Bruno subalgebras of the Hopf algebra of planar trees from combinatorial DysonSchwinger equations. Adv. Math., 218(1):136-162, 2008.

[Fra08] A. Frabetti. Groups of tree-expanded series. J. Algebra, 319(1):377-413, 2008.

$\left[\mathrm{GKL}^{+}\right.$95] I. M. Gelfand, D. Krob, A. Lascoux, B. Leclerc, V. S. Retakh, and J.-Y. Thibon. Noncommutative symmetric functions. Adv. Math., 112(2):218-348, 1995.

[HNT05] F. Hivert, J.-C. Novelli, and J.-Y. Thibon. The Algebra of Binary Search Trees. Theor. Comput. Sci., 339(1):129-165, 2005.

[JR79] S. A. Joni and G.-C. Rota. Coalgebras and bialgebras in combinatorics. Stud. Appl. Math., 61:93-139, 1979.

[KLT97] D. Krob, B. Leclerc, and J.-Y. Thibon. Noncommutative symmetric functions. II. Transformations of alphabets. Int. J. Algebr. Comput., 7(2):181-264, 1997.

[Laf11] Y. Lafont. Diagram rewriting and operads. Sémin. Congr., 26:163-179, 2011.

[Lei04] T. Leinster. Higher operads, higher categories, volume 298 of London Mathematical Society Lecture Note Series. Cambridge University Press, Cambridge, 2004.

[LR98] J.-L. Loday and M. O. Ronco. Hopf Algebra of the Planar Binary Trees. Adv. Math., 139:293-309, 1998.

[LV12] J.-L. Loday and B. Vallette. Algebraic Operads, volume 346 of Grundlehren der mathematischen Wissenschaften. Springer, 2012. 
[Mac95] I. G. Macdonald. Symmetric functions and Hall polynomials. Oxford University Press, second edition, 1995.

[Mar08] M. Markl. Operads and PROPs. In Handbook of Algebra, volume 5, pages 87-140. Elsevier/NorthHolland, Amsterdam, 2008.

[May72] J. P. May. The geometry of iterated loop spaces. Lect. Notes Math., 271, 1972.

[ML65] S. Mac Lane. Categorical algebra. Bull. Amer. Math. Soc., 71:40-106, 1965.

[ML14] M. Méndez and J. Liendo. An antipode formula for the natural Hopf algebra of a set operad. Adv. Appl. Math., 53:112-140, 2014.

[MR95] C. Malvenuto and C. Reutenauer. Duality between quasi-symmetric functions and Solomon descent algebra. J. Algebra, 177:967-982, 1995.

[PR95] S. Poirier and C. Reutenauer. Algèbres de Hopf de tableaux. Ann. Sci. Math. Québec, 19(1):79-90, 1995.

$\left[\mathrm{S}^{+} 14\right] \quad$ W. A. Stein et al. Sage Mathematics Software (Version 6.1.1). The Sage Development Team, 2014. http://www. sagemath.org.

[SCc08] The Sage-Combinat community. Sage-Combinat: enhancing Sage as a toolbox for computer exploration in algebraic combinatorics, 2008. http://combinat.sagemath.org.

[Slo] N. J. A. Sloane. The On-Line Encyclopedia of Integer Sequences. https://oeis. org/.

[Tak71] M. Takeuchi. Free Hopf algebras generated by coalgebras. J. Math. Soc. Japan, 23(4):561-582, 1971.

[vdL04] P. van der Laan. Operads. Hopf algebras and coloured Koszul duality. PhD thesis, Universiteit Utrecht, 2004.

[Vie86] X. Viennot. Heaps of pieces. I. Basic definitions and combinatorial lemmas. In Combinatoire énumérative, volume 1234 of Lect. Notes Math., pages 321-350. Springer, 1986.

Laboratoire d'Informatique, du Traitement de l'Information et des Systèmes, Université de Rouen, Avenue de L'Université, 76801 Saint-Étienne-du-Rouvray cedex, France.

E-mail address: jean-paul.bultel@univ-rouen.fr

Laboratoire d'Informatique Gaspard-Monge, Université Paris-Est Marne-la-Vallée, 5 boulevard Descartes, Champs-sur-Marne, 77454 Marne-la-Vallée cedex 2, France.

E-mail address: samuele.giraudo@u-pem.fr 